\title{
Distribution, Enrichment and Modes of Occurrence of Arsenic in Chinese Coals
}

\author{
Jiangfeng Guo ${ }^{1,2, *}$ D, Duoxi Yao ${ }^{1, *}$, Ping Chen ${ }^{1}$, Jian Chen ${ }^{1}$ and Fengjun Shi ${ }^{2}$ \\ 1 School of Earth and Environment, Anhui University of Science and Technology, Huainan 232001, China; \\ pchen87@163.com (P.C.); cscchenjian@163.com (J.C.) \\ 2 School of Mechanical Engineering, Jiangsu College of Engineering and Technology, Nantong 226007, China; \\ shifengjunjudy@hotmail.com \\ * Correspondence: jfguo@163.com (J.G.); dxyao@aust.edu.cn (D.Y.); \\ Tel.: +86-554-6631-870 (D.Y.); +86-513-8105-0306 (J.G.)
}

Received: 10 April 2017; Accepted: 30 June 2017; Published: 3 July 2017

\begin{abstract}
Arsenic is one of the toxic trace elements in coals, which is harmful to both the ecological environment and human health. Based on published literature and the data obtained by our research group, a total of 5314 As concentrations of Chinese coals were analyzed. The arithmetic mean of arsenic content in Chinese coals is $6.97 \mathrm{mg} / \mathrm{kg}$. Choosing the percentage of provincial coal resources in national coal resources as the weighting factor, the weighted average of arsenic content in Chinese coals is $5.33 \mathrm{mg} / \mathrm{kg}$. The content of arsenic in Chinese coals increases from the north to the south. High arsenic content in coal primarily occurs in southwestern Yunnan and certain coalfields in the Guizhou Province. Additionally, arsenic is enriched in the coals from some regions, i.e., the western Yunnan, Guangxi, Tibet, southwestern Liaoning, Jilin, and Henan. The arsenic content in coals of different coal-forming periods shows an overall regularity: Paleogene and Neogene $>$ Late Triassic > Late Permian > Late Jurassic and Early Cretaceous > Early and Middle Jurassic $>$ Late Carboniferous and Early Permian. The modes of occurrence of arsenic in coals include sulfide-association, organic-association, arsenate-association, silicate-association, and soluble- and exchangeable-association. Generally, arsenic in Chinese coals exists predominantly in arsenic-bearing pyrite. Meanwhile, the organic arsenic content is relatively high in coal samples with a lower $(<5.5 \mathrm{mg} / \mathrm{kg})$ arsenic content and a low or medium ash yield $(<30 \%)$.
\end{abstract}

Keywords: arsenic; Chinese coals; distribution; enrichment; modes of occurrence

\section{Introduction}

In 2016, China's coal production was 3.41 billion tons, which was $7.9 \%$ lower than 2015 , accounting for $46.1 \%$ of the total world coal production. Additionally, the coal consumption was 3.82 billion tons, $50.6 \%$ of the world consumption [1,2]. The share of coal as China's primary energy source reached $62.0 \%$ in 2016 [2], and coal will locate in a dominant position of Chinese energy structure in the foreseeable future.

The toxic trace elements in coals can migrate into the atmosphere, hydrosphere and soil, causing environmental pollution and even harmful impacts on human health during coal utilization [3]. As one of the most toxic trace elements in coals, arsenic could be released into the atmosphere during coal combustion and preferentially enriched in fly ash [4]. The arsenic-rich fly ash could remain in the air for a long time and enter the human body through the respiratory system [5]. Finally, the fly ash precipitates on the earth's surface, resulting water and soil pollution. The amount of arsenic entering the soil cycle in the form of fly ash reached 2200 tons every year [6]. Global arsenic emission from coal combustion was 6240 tons per year [7]. The atmospheric arsenic emissions from coal combustion reached 1564 tons in China in 2005 [8]. Arsenic can also be enriched in coal-hosted 
rare-metal deposits and their corresponding coal combustion products, which could have adverse effects for both environment and human health [9-12].

The earliest study on arsenic in world coals could be traced back to the 1934 conducted by Goldschmidt and Peters [13]. Before determined by inductively coupled plasma mass spectrometry (ICP-MS), coal samples should be digested. The microwave digestion program, related to coal and coal-related materials, was outlined by Dai et al. [14]. And it was recently determined by ICP-MS using collision cell technology (CCT) in order to avoid disturbance of polyatomic ions [15,16]. Valkovic [17], Yudovich et al. [18], and Ketris and Yudovich [19] estimated the average content of arsenic in coal. The modes of occurrence of arsenic in coals, migration and transformation mechanisms were discussed [13,20,21]. Finkelman [22] assigned the confidence level for arsenic occurrence in coal in pyrite as 8 . The methods for the modes of occurrence of trace elements in coals can be divided into direct and indirect methods, i.e., microscopic and spectral analysis, float and sink analysis, sequential chemical extraction, low temperature ashing $+\mathrm{X}$-ray diffraction, and statistical analysis [23]. The Mega-pixel Synchrotron X-ray Fluorescence (MSXRF), X-ray Absorption Near Edge Structure (XANES), and Extended X-ray Absorption Fine Structure (EXAFS) have recently contributed to the modes of occurrence of arsenic in coals [24]. The sulfides-associated As dominate at high As content, while As org dominates at a low As level [25]. Regarding pyrite-hosted arsenic in coal, it occurs as a solid solution [26].

In 1964, villagers in the Zhijin County of Guizhou Province suffered from chronically arsenism due to the high arsenic level in coals, attracting much attention on the arsenic in Chinese coals [27-32]. Besides Guizhou, the arsenism also occurred in the Yunnan and Shaanxi Provinces [33-36]. However, not all the Zhijin coals are rich in arsenic [31,37,38]. The mechanism of arsenic enrichment in Chinese coals was fully discussed by Zhao [30], Ren et al. [39], and Dai et al. [40]. There are five types, i.e., source-rock-controlled, marine-environment-controlled, hydrothermal-fluid-controlled, groundwater-controlled, and volcanic-ash-controlled [40].

Due to the complex geologic conditions, multiple coal-forming periods, and substantial epigenetic changes of Chinese coals, a comprehensive review on arsenic in Chinese coals is requisite. Thus, the average arsenic concentration of Chinese coals, spatial distribution, and modes of occurrence will be reviewed. In this paper, based on the published literature and the data of our research group, the arsenic in Chinese coals are completely reviewed, including arithmetic and weighted average of arsenic contents, distribution, abnormal enrichment, and modes of occurrence.

\section{Content of Arsenic in Coals}

\subsection{Content of Arsenic in World Coals}

In some countries, such as the United State of America, former Soviet Union, Australia, and Czech Republic, the geochemistry of arsenic in coal has completely investigated. The content of arsenic in American coal was $24.0 \mathrm{mg} / \mathrm{kg}$ (7676 samples), with a maximum of $2200 \mathrm{mg} / \mathrm{kg}$ [41]. The content of arsenic in the former Soviet Union coals was $25 \mathrm{mg} / \mathrm{kg}$ [42]. It reached $32.7 \mathrm{mg} / \mathrm{kg}$ in the Donetz coalfield [43]. Swaine [13,44] calculated that the arsenic contents in Australian coals were 1.50 and $2.00 \mathrm{mg} / \mathrm{kg}$, respectively. Based on 9172 samples in the Bohemia basin, the arsenic content of coals from the northern Czech Republic ranged from 0.10 to $757 \mathrm{mg} / \mathrm{kg}$, with an arithmetic mean of 39.9 $\mathrm{mg} / \mathrm{kg}$ [45]. Furthermore, Pesek et al. [46] calculated that the content of arsenic in Czech coal increased from $<0.10$ to $2020 \mathrm{mg} / \mathrm{kg}$, with an average of $209 \mathrm{mg} / \mathrm{kg}$ (23,601 coal samples). Yudovich and Ketris [25] estimated the average arsenic content of world coals and gave arsenic abundances in brown and bituminous coals of $7.6 \pm 1.3 \mathrm{mg} / \mathrm{kg}$ and $9.0 \pm 0.7 \mathrm{mg} / \mathrm{kg}$, respectively. According to the panel on the trace element geochemistry of coal resource development related to health (PECH) statistics [47], the average content of arsenic in world coals was $5 \mathrm{mg} / \mathrm{kg}$.

During the last two decades, several As-enriched coal deposits were reported from Turkey. The As enrichments in Turkish coal deposits was mostly related to the synchronous volcanic activity and leached surface waters [48-51]. The arsenic accumulation caused by synchronous volcanic activity 
was called "Turkish (volcanogenic) type" [25]. Furthermore, in certain places, epigenetic hydrothermal mineralization-related influence also elevated As-concentrations (up to $3854 \mathrm{mg} / \mathrm{kg}$ in Gediz coal) [52], and the multistage As-enrichments (up to $984 \mathrm{mg} / \mathrm{kg}$ ) were reported [53].

\subsection{Content of Arsenic in Chinese Coals}

Chen et al. [27], analyzed 107 samples and reported the content of arsenic in Chinese coals, ranging from 0.32 to $97.8 \mathrm{mg} / \mathrm{kg}$. Sun and Jervis [54] used 15 coal samples to calculate an arsenic content (0.06 to $124 \mathrm{mg} / \mathrm{kg})$. Dou et al. [55] analyzed 732 samples in the Shenfu-Dongsheng mining area, concluding that the arsenic content in coal was in the range of $0.04-78.0 \mathrm{mg} / \mathrm{kg}$, with an arithmetic mean of $1.77 \mathrm{mg} / \mathrm{kg}$. Ren et al. [56] gave an arsenic content of 132 coal samples with $0.21-32,000 \mathrm{mg} / \mathrm{kg}$ (arithmetic mean of $276.6 \mathrm{mg} / \mathrm{kg}$ ), which was ascribed to the introduction of some high arsenic coal samples from the southwestern China. There are significant variations of arsenic content in Chinese coals, especially some southwestern coal mines with elevated arsenic. For example, Ding et al. [32] reported that the arsenic in the Anlong coal from Guizhou Province reached 35,000 mg/kg, the greatest value around the world. Fortunately, the reserve of such extremely high-As coals is extraordinarily small. In view of its severe negative impact on environment, the local government has shut down these coal mines. It was not representative of mineable coalfields in China. Ren et al. [39] introduced a "reserves weight" method to recount 3453 Chinese coal samples and gave a new arithmetic mean of $3.80 \mathrm{mg} / \mathrm{kg}$. Chen et al. [57] excluded the abnormally high arsenic coal samples in the calculation of common arsenic content in Chinese coals, ranging from 0.80 to $20.0 \mathrm{mg} / \mathrm{kg}$ (arithmetic mean of $4.00 \mathrm{mg} / \mathrm{kg}$ ). Dai et al. [40] updated the value by analyzing 3386 coal samples, with an average of $3.79 \mathrm{mg} / \mathrm{kg}$.

The high-arsenic coal occurs in southwestern Guizhou Province, resulting in a serious endemic arsenism caused by the domestic arsenic-rich coal combustion. More than 3000 patients in the mountainous region of southwestern Guizhou suffered from arsenosis [58]. The highest values given by Ding et al. (35,000 mg/kg) [32] and Zhao et al. (32,000 mg/kg) [59] were from southwestern Guizhou, so the general impression to many people is that all the coals in Guizhou are characterized by high arsenic. In fact, abnormal high-arsenic coal samples (such as 35,000 and 32,000 mg/kg) were from a small coal mine nearby, not a workable mine. The As-rich coals are not ubiquitous in the southwestern Guizhou [40], and the As-rich coals locate in a restricted area [60,61]. On the other hand, preventive measures for endemic arsenosis in Guizhou have achieved desired results [40].

In this paper, data from the published literature and our research group, with a total samples of 5314 from 30 provinces in China, are statistically analyzed. The arithmetic mean arsenic content of Chinese coals is $6.97 \mathrm{mg} / \mathrm{kg}$, including many coal samples with a high arsenic content from the southwestern China, e.g., Lincang coals (117 mg/kg) in Yunnan Province [62] and Zhenfeng coals $(54.8 \mathrm{mg} / \mathrm{kg})$ in Guizhou Province [63]. The arsenic concentrations in Chinese coals are presented in Table 1.

The geographic distribution of arsenic in Chinese coal is extremely uneven, inducing a simple calculation of arithmetic mean cannot represent the common arsenic content of Chinese coals. Selecting the percentages of each province's coal resources in the Chinese total coal resources as weighting factors, the weighted averaging arsenic content in coals is calculated. This method can eliminate the difference between samples and geologic conditions caused by uneven sampling. Based on the predicted coal resources reported by the China Coal Geology Bureau [64], the resources weighted average arsenic content is $5.33 \mathrm{mg} / \mathrm{kg}$, slightly higher than the data given by Dai et al. [40] and Ren et al. [39]. The results are listed in Tables 2 and 3, respectively. The arithmetic arsenic content in Chinese coals is far lower than that of American and Czech coals, but higher than that of Australian coals. However, with respect to the weighted arsenic content of Chinese coals, there is a little difference between China and world coals of $5 \mathrm{mg} / \mathrm{kg}$ [47]. 
Table 1. Arsenic concentration in Chinese coals (mg/kg). Data are quoted from references of $[30,31,37-40,58,62,63,65-144]$

\begin{tabular}{|c|c|c|c|c|c|c|c|c|c|}
\hline Province/Coal Mine & $\begin{array}{l}\text { Arithmetic } \\
\text { Mean }\end{array}$ & $\begin{array}{c}\text { Sample } \\
\text { Number }\end{array}$ & $\begin{array}{c}\text { Coal-Forming } \\
\text { Period }\end{array}$ & Data Source & Province/Coal Mine & $\begin{array}{c}\text { Arithmetic } \\
\text { Mean }\end{array}$ & $\begin{array}{c}\text { Sample } \\
\text { Number }\end{array}$ & $\begin{array}{c}\text { Coal-Forming } \\
\text { Period }\end{array}$ & Data Source \\
\hline Huainan, Anhui & 3.57 & 22 & C-P & Ge [65] & Daqingshan, Inner Mongolia & 0.97 & 9 & C-P & Dai et al. [113] \\
\hline Huaibei, Anhui & 0.6 & 7 & C-P & Tang et al. [66] & Jungar Haerwusu, Inner Mongolia & 0.6 & 29 & C-P & Dai et al. [114] \\
\hline Huaibei, Anhui & 2.9 & 5 & C-P & Tang et al. [66] & Jungar Chuancaogedan 5, Inner Mongolia & 0.28 & 15 & C-P & Yang et al. [115] \\
\hline Huainan Xinzhuangzi, Anhui & 10.7 & 2 & C-P & Tang et al. [66] & Jungar Chuancaogedan 6, Inner Mongolia & 0.56 & 7 & C-P & Dai et al. [116] \\
\hline Huainan Xinzhuangzi, Anhui & 1.8 & 9 & C-P & Tang et al. [66] & Jungar Heidaigou, Inner Mongolia & 0.99 & 30 & $\mathrm{P}_{2}$ & Dai et al. [117] \\
\hline Huainan Liyi, Anhui & 11.5 & 5 & $\mathrm{C}-\mathrm{P}$ & Tang et al. [66] & Yimin, Inner Mongolia & 3 & 1 & $\mathrm{~J}_{3}-\mathrm{K}_{1}$ & Ren et al. [39] \\
\hline Huainan Panyi, Anhui & 0.4 & 6 & C-P & Tang et al. [66] & Dayan, Inner Mongolia & 1 & 2 & $\mathrm{~J}_{3}-\mathrm{K}_{1}$ & Ren et al. [39] \\
\hline Huaibei Mengzhuang, Anhui & 3.66 & 15 & $\mathrm{C}-\mathrm{P}$ & Zhao and Wu [67] & Huolinhe, Inner Mongolia & 12.88 & 3 & $\mathrm{~J}_{3}-\mathrm{K}_{1}$ & Bai [118] \\
\hline Huainan, Anhui & 2.02 & 17 & C-P & Chen et al. [68] & Jalainur, Inner Mongolia & 2.59 & 3 & $\mathrm{~J}_{3}-\mathrm{K}_{1}$ & Ren et al. [39] \\
\hline Huainan, Anhui & 4.21 & 24 & $\mathrm{C}-\mathrm{P}$ & Tong et al. [69] & Yuanbaoshan, Inner Mongolia & 2.95 & 2 & $\mathrm{~J}_{3}-\mathrm{K}_{1}$ & Ren et al. [39] \\
\hline Huainan Zhangji, Anhui & 9.07 & 144 & C-P & Li et al. [70] & Xidayao, Inner Mongolia & 3.4 & 5 & $\mathrm{~J}_{1-2}$ & Ren et al. [39] \\
\hline Huaibei, Anhui & 1.51 & 12 & C-P & Chen et al. [71] & Ordos Basin, Inner Mongolia & 16.3 & 138 & $J_{1-2}$ & Li et al. [119] \\
\hline Huainan, Anhui & 1.58 & 24 & $\mathrm{C}-\mathrm{P}$ & Chen et al. [71] & Shengli, Inner Mongolia & 8.46 & 30 & $\mathrm{~J}_{3}-\mathrm{K}_{1}$ & Dai et al. [120] \\
\hline Huainan, Anhui & 4.43 & 22 & C-P & Chen and Tang [72] & Shenfu-Dongsheng & 1.77 & 732 & $\mathrm{~J}_{1-2}$ & Tang et al. [66] \\
\hline Anqing and Tongling, Anhui & 10.16 & 36 & $\mathrm{P}_{2}$ & Qian and Yang [73] & Shenfu-Dongsheng & 0.42 & 5 & $J_{1-2}$ & Tang et al. [66] \\
\hline Beijing & 1.62 & 3 & $\mathrm{C}-\mathrm{P}$ & Ren et al. [39] & Jiangoushan, Ningxia & 0.55 & 1 & $\mathrm{C}-\mathrm{P}$ & Ren et al. [39] \\
\hline Daanshan, Beijing & 1 & 1 & $\mathrm{~J}_{1-2}$ & Ren et al. [39] & Shizuishan, Ningxia & 1.57 & 8 & C-P & Dai [102] \\
\hline Changhe, Chongqing & 10.02 & 5 & $\mathrm{P}_{3}$ & Wang [74] & Shitanjing, Ningxia & 0.97 & 11 & C-P & Dai [102] \\
\hline Songzao, Chongqing & 3.17 & 32 & $\mathrm{P}_{2}$ & Ren et al. [39] & Tongxin, Ningxia & 1 & 3 & $\mathrm{C}-\mathrm{P}$ & Dai [102] \\
\hline Nantong, Chongqing & 3.08 & 21 & $\mathrm{P}_{2}$ & Ren et al. [39] & Rujigou, Ningxia & 0.74 & 5 & $\mathrm{~J}_{1-2}$ & Song [121] \\
\hline Zhongliangshan, Chongqing & 4.12 & 2 & $\mathrm{P}_{2}$ & Ren et al. [39] & Ciyaobao, Ningxia & 10.62 & 2 & $\mathrm{~J}_{1-2}$ & Li et al. [119] \\
\hline Tianfu, Chongqing & 4.4 & 3 & $\mathrm{P}_{2}$ & Ren et al. [39] & Yuka, Qinghai & 1.34 & 1 & $J_{1-2}$ & Ren et al. [39] \\
\hline Chuandong, Chongqing & 5.24 & 5 & $\mathrm{P}_{2}$ & Ren et al. [39] & Mole, Qinghai & 2.77 & 1 & $J_{1-2}$ & Ren et al. [39] \\
\hline Chuandongnan, Chongqing & 7.4 & 2 & $\mathrm{P}_{2}$ & Ren et al. [39] & Jiangcang, Qinghai & 3.05 & 1 & $\mathrm{~J}_{1-2}$ & Ren et al. [39] \\
\hline Chongqing & 3.07 & 1 & $\mathrm{P}_{2}$ & Luo et al. [75] & Datong, Qinghai & 3.62 & 1 & $\mathrm{~J}_{1-2}$ & Ren et al. [39] \\
\hline Donglin and Nantong, Chongqing & 2.547 & 32 & $\mathrm{P}_{2}$ & this study & Tibet Plateau & 0.788 & 16 & $\mathrm{~J}_{1-2}$ & Dai et al. [122] \\
\hline Songzao, Chongqing & 9.14 & 4 & $\mathrm{P}_{2}$ & Dai et al. [76] & Huangxian, Shandong & 2.9 & 2 & $\mathrm{E}-\mathrm{N}$ & Ren et al. [39] \\
\hline Songzao, Chongqing & 8.06 & 4 & $\mathrm{P}_{2}$ & Dai et al. [76] & Feicheng and Xinwen, Shandong & 11.4 & 17 & $\mathrm{C}-\mathrm{P}$ & Zen et al. [123] \\
\hline Songzao, Chongqing & 5.84 & 5 & $\mathrm{P}_{2}$ & Dai et al. [76] & Feicheng and Xinwen, Shandong & 1.57 & 6 & C-P & Zen et al. [123] \\
\hline Songzao, Chongqing & 9.11 & 4 & $\mathrm{P}_{2}$ & Dai et al. [76] & Zibo, Shandong & 7.9 & 1 & C-P & Tang et al. [66] \\
\hline Songzao, Chongqing & 5.42 & 4 & $\mathrm{P}_{2}$ & Dai et al. [76] & Chaili, Shandong & 3.5 & 1 & $\mathrm{C}-\mathrm{P}$ & Tang et al. [66] \\
\hline Songzao, Chongqing & 9.52 & 4 & $\mathrm{P}_{2}$ & Dai et al. [76] & Taozhuang, Shandong & 0.45 & 2 & C-P & Tang et al. [66] \\
\hline Songzao, Chongqing & 25.7 & 5 & $\mathrm{P}_{2}$ & Dai et al. [76] & Zaozhuang, Shandong & 5.6 & 10 & $\mathrm{C}-\mathrm{P}$ & Tang et al. [66] \\
\hline Chuandong, Chongqing & 9.2 & 10 & $\mathrm{P}_{3}$ & Ren et al. [39] & Jibei, Shandong & 19.62 & 56 & C-P & $\mathrm{Hu}[124]$ \\
\hline Yongrong, Chongqing & 10.43 & 17 & $\mathrm{P}_{3}$ & Ren et al. [39] & Zaozhuang, Shandong & 7.28 & 21 & C-P & Chen et al. [71] \\
\hline Changhebian, Chongqing & 10.03 & 5 & $\mathrm{P}_{3}$ & Wang et al. [77] & Jining, Shandong & 2.34 & 38 & C-P & Liu [125] \\
\hline Moxinpo, Chongqing & 2.27 & 4 & $\mathrm{P}_{2}$ & Dai et al. [78] & Juye, Shandong & 2.25 & 49 & $\mathrm{C}-\mathrm{P}$ & Zhao et al. [89] \\
\hline Moxinpo, Chongqing & 10.7 & 4 & $\mathrm{P}_{2}$ & Dai et al. [78] & Xinwen, Shandong & 1.67 & 7 & C-P & Zhang [126] \\
\hline Yongan, Fujian & 12 & 3 & $\mathrm{P}_{2}$ & Lu et al. [79] & Feicheng, Shandong & 2.3 & 5 & C-P & Zen et al. [123] \\
\hline Fujian & 7.5 & 2 & & Cui and Chen [80] & Tengxian, Shandong & 0.8 & 1 & C-P & Ren et al. [39] \\
\hline Zhangye, Gansu & 1.38 & 4 & $\mathrm{~J}_{1-2}$ & Ren et al. [39] & Taozao, Shandong & 3.45 & 13 & $\mathrm{C}-\mathrm{P}$ & Chen and Tang [72 \\
\hline Ankou, Gansu & 1.5 & 2 & $J_{1-2}$ & Ren et al. [39] & Yanzhou, Shandong & 1.79 & 6 & C-P & Liu et al. [127] \\
\hline Dayou, Gansu & 6.43 & 2 & $\mathrm{~J}_{1-2}$ & Ren et al. [39] & Liaocheng, Shandong & 7.9 & 1 & C-P & Ren et al. [39] \\
\hline
\end{tabular}


Table 1. Cont

\begin{tabular}{|c|c|c|c|c|c|c|c|c|c|}
\hline Province/Coal Mine & $\begin{array}{l}\text { Arithmetic } \\
\text { Mean }\end{array}$ & $\begin{array}{l}\text { Sample } \\
\text { Number }\end{array}$ & $\begin{array}{c}\text { Coal-Forming } \\
\text { Period }\end{array}$ & Data Source & Province/Coal Mine & $\begin{array}{l}\text { Arithmetic } \\
\text { Mean }\end{array}$ & $\begin{array}{c}\text { Sample } \\
\text { Number }\end{array}$ & $\begin{array}{c}\text { Coal-Forming } \\
\text { Period }\end{array}$ & Data Source \\
\hline Huating, Gansu & 3.33 & 2 & $\mathrm{~J}_{1-2}$ & Ren et al. [39] & Zibo and Taozhuang, Shandong & 4.12 & 14 & C-P & Chen et al. [57] \\
\hline Baojishan, Gansu & 3.47 & 2 & $J_{1-2}$ & Ren et al. [39] & Hengqu, Shanxi & 11.87 & 3 & $\mathrm{E}-\mathrm{N}$ & Tang et al. [66] \\
\hline Yaojie, Gansu & 3 & 1 & $\mathrm{~J}_{1-2}$ & Ren et al. [39] & Shanxi & 1.37 & 57 & $\mathrm{C}-\mathrm{P}$ & Tang et al. [66] \\
\hline Wangjiashan, Gansu & 2 & 1 & $\mathrm{~J}_{1-2}$ & Ren et al. [39] & Hunyuan, Shanxi & 4.7 & 1 & C-P & Zhang et al. [107] \\
\hline Guangzhou, Guangdong & 13.9 & 1 & $\mathrm{P}_{2}$ & Lu et al. [79] & Pingshuo, Shanxi & 6.9 & 8 & $\mathrm{C}-\mathrm{P}$ & Zhuang et al. [128] \\
\hline Xingmei, Guangdong & 8 & 1 & $\mathrm{P}_{2}$ & Ren et al. [39] & Pingshuo, Shanxi & 3.9 & 2 & $\mathrm{C}-\mathrm{P}$ & Zhao et al. [89] \\
\hline Guangdong & 9.6 & 33 & & Cui and Chen [80] & Shuozhou Pinglu, Shanxi & 0.2 & 1 & C-P & Zhang et al. [107] \\
\hline Baise, Guangxi & 25.4 & 2 & $\mathrm{E}-\mathrm{N}$ & Ren et al. [39] & Zuoquan, Shanxi & 6.8 & 1 & $\mathrm{C}-\mathrm{P}$ & Zhang et al. [107] \\
\hline Nanning, Guangxi & 19.6 & 2 & $\mathrm{E}-\mathrm{N}$ & Ren et al. [39] & Yangquan, Shanxi & 0.7 & 1 & C-P & Zhang et al. [107] \\
\hline Hongmao, Guangxi & 3.84 & 1 & $\mathrm{C}-\mathrm{P}$ & Ren et al. [39] & Yangquan, Shanxi & 1.23 & 2 & $\mathrm{C}-\mathrm{P}$ & Zhao et al. [89] \\
\hline Heshan, Guangxi & 3.36 & 12 & $\mathrm{P}_{2}$ & Li et al. [81] & Lingshi, Shanxi & 1 & 1 & $\mathrm{C}-\mathrm{P}$ & Zhang et al. [107] \\
\hline Fusui, Guangxi & 8.59 & 10 & $\mathrm{P}_{2}$ & Dai et al. [82] & Xishan, Shanxi & 3.4 & 1 & C-P & Tang et al. [66] \\
\hline Heshan, Guangxi & 13.58 & 12 & $\mathrm{P}_{2}$ & Shao et al. [83] & Fenxi, Shanxi & 0.8 & 3 & C-P & Zhang et al. [107] \\
\hline Heshan, Guangxi & 11.8 & 4 & $\mathrm{P}_{2}$ & Dai et al. [84] & Huoxi, Shanxi & 2 & 7 & C-P & Tang et al. [66] \\
\hline Heshan, Guangxi & 3.37 & 6 & $\mathrm{P}_{2}$ & Dai et al. [84] & Pingshuo, Shanxi & 2.96 & 6 & $\mathrm{C}-\mathrm{P}$ & Zhao [30] \\
\hline Heshan, Guangxi & 5.31 & 2 & $\mathrm{P}_{2}$ & Dai et al. [84] & Pingshuo, Shanxi & 0.4 & 57 & C-P & Ren et al. [39] \\
\hline Yishan, Guangxi & 8.3 & 22 & $\mathrm{P}_{3}$ & Dai et al. [85] & Hedong, Shanxi & 0.99 & 29 & C-P & $\mathrm{Li}[111]$ \\
\hline Shuicheng, Guizhou & 0.92 & 3 & $\mathrm{P}_{2}$ & Zen et al. [86] & Huozhou, Shanxi & 1.81 & 9 & $\mathrm{C}-\mathrm{P}$ & Chen and Tang [72] \\
\hline Shuicheng, Guizhou & 6.26 & 3 & $\mathrm{P}_{2}$ & Zen et al. [86] & Xishan, Shanxi & 4.27 & 19 & $\mathrm{C}-\mathrm{P}$ & Ge [129] \\
\hline Liuzhi Shuicheng, Guizhou & 8 & 45 & $\mathrm{P}_{2}$ & Zhuang et al. [87] & Yangquan, Shanxi & 1.17 & 7 & C-P & Zhao [30] \\
\hline Lindong, Guizhou & 7.6 & 32 & $\mathrm{P}_{2}$ & $\mathrm{Ni}$ et al. [88] & Jincheng, Shanxi & 2.22 & 5 & C-P & Wang [130] \\
\hline Nayong, Guizhou & 1.26 & 1 & $\mathrm{P}_{2}$ & Zhao et al. [89] & Lu'an, Shanxi & 0.95 & 1 & $\mathrm{C}-\mathrm{P}$ & Bai [118] \\
\hline Zhijin Shuchang, Guizhou & 26.5 & 4 & $\mathrm{P}_{2}$ & An et al. [90] & Ningwu, Shanxi & 0.29 & 1 & $\mathrm{C}-\mathrm{P}$ & Ren et al. [39] \\
\hline Zhijin Xingzhai, Guizhou & 2.5 & 3 & $\mathrm{P}_{2}$ & An et al. [90] & Xuangang, Shanxi & 3.43 & 1 & C-P & Ren et al. [39] \\
\hline Xingren Jiaole, Guizhou & 10.8 & 18 & $\mathrm{P}_{2}$ & Zhou et al. [91] & Datong, Shanxi & 8 & 6 & C-P & Ren et al. [39] \\
\hline Dafang, Guizhou & 5.79 & 71 & $\mathrm{P}_{2}$ & Dai et al. [92] & Hongdong, Shanxi & 2.87 & 5 & C-P & Ren et al. [39] \\
\hline Panxian, Guizhou & 3.68 & 16 & $\mathrm{P}_{2}$ & Feng et al. [93] & Changzhi, Shanxi & 2 & 1 & $\mathrm{C}-\mathrm{P}$ & Ren et al. [39] \\
\hline Shuicheng, Guizhou & 7.15 & 25 & $\mathrm{P}_{2}$ & Feng et al. [93] & Gujiao, Shanxi & 1.47 & 3 & $\mathrm{C}-\mathrm{P}$ & Ge [129] \\
\hline Guiyang, Guizhou & 7.27 & 3 & $\mathrm{P}_{2}$ & Feng et al. [93] & Gujiao, Shanxi & 6.73 & 2 & C-P & $\mathrm{Ge}[129]$ \\
\hline Liuzhi, Guizhou & 8.52 & 15 & $\mathrm{P}_{2}$ & Feng et al. [93] & Pingshuo, Shanxi & 3.91 & 2 & C-P & Zhao et al. [89] \\
\hline Nayong, Guizhou & 2.59 & 6 & $\mathrm{P}_{2}$ & Zhou [31] & Fenxi, Shanxi & 1.1 & 1 & C-P & Zhang et al. [107] \\
\hline Zhijin, Guizhou & 5.27 & 59 & $\mathrm{P}_{2}$ & Zhou [31] & Xishan, Shanxi & 0.75 & 2 & C-P & Zhang et al. [107] \\
\hline Zhijin, Guizhou & 4.88 & 15 & $\mathrm{P}_{2}$ & Dai et al. [38] & Shanxi & 2.07 & 89 & $\mathrm{C}-\mathrm{P}$ & Zhang et al. [131] \\
\hline Zhijin, Guizhou & 1.1 & 1 & $\mathrm{P}_{2}$ & Dai et al. [37] & Fanshi and Yuanqu, Shanxi & 13.5 & 4 & $\mathrm{P}_{3}$ & Zhang et al. [131] \\
\hline Qinglong, Guizhou & 39.15 & 4 & $\mathrm{P}_{2}$ & Zhang [63] & Datong, Shanxi & 7.61 & 30 & $\mathrm{~J}_{1-2}$ & Zhuang et al. [128] \\
\hline Xishui, Guizhou & 3.19 & 7 & $\mathrm{P}_{2}$ & Ren et al. [39] & Datong, Shanxi & 8.5 & 3 & $\mathrm{~J}_{1-2}$ & Zhang et al. [107] \\
\hline Bijie, Guizhou & 5.54 & 4 & $\mathrm{P}_{2}$ & Dai et al. [94] & Datong, Shanxi & 4.79 & 8 & $\mathrm{~J}_{1-2}$ & Zhuang et al. [100] \\
\hline Guiding, Guizhou & 6.06 & 1 & $\mathrm{P}_{2}$ & Lei [95] & Datong, Shanxi & 3.84 & 8 & $\mathrm{~J}_{1-2}$ & Chen et al. [71] \\
\hline southwest of Guizhou & 10.72 & 36 & $\mathrm{P}_{2}$ & Zhang [63] & Datong, Shanxi & 12.3 & 17 & $\mathrm{~J}_{1-2}$ & Zhang et al. [131] \\
\hline Guizhou & 12.51 & 1 & $\mathrm{P}_{2}$ & Luo et al. [75] & Pubai, Shaanxi & 2.14 & 1 & $\mathrm{C}-\mathrm{P}$ & Ren et al. [39] \\
\hline Qinglong, Guizhou & 1.58 & 15 & $\mathrm{P}_{2}$ & Li and Tang [96] & Chenghe, Shaanxi & 1.36 & 1 & $\mathrm{C}-\mathrm{P}$ & Ren et al. [39] \\
\hline
\end{tabular}


Table 1. Cont

\begin{tabular}{|c|c|c|c|c|c|c|c|c|c|}
\hline Province/Coal Mine & $\begin{array}{c}\text { Arithmetic } \\
\text { Mean }\end{array}$ & $\begin{array}{c}\text { Sample } \\
\text { Number }\end{array}$ & $\begin{array}{c}\begin{array}{c}\text { Coal-Forming } \\
\text { Period }\end{array} \\
\end{array}$ & Data Source & Province/Coal Mine & $\begin{array}{c}\text { Arithmetic } \\
\text { Mean }\end{array}$ & $\begin{array}{c}\text { Sample } \\
\text { Number }\end{array}$ & $\begin{array}{c}\begin{array}{c}\text { Coal-Forming } \\
\text { Period }\end{array} \\
\end{array}$ & Data Source \\
\hline Liupanshui, Guizhou & 5.87 & 62 & $\mathrm{P}_{2}$ & Guo et al. [97] & Hancheng, Shaanxi & 1 & 1 & $\mathrm{C}-\mathrm{P}$ & Ren et al. [39] \\
\hline $\begin{array}{l}\text { Xingren, Zhijin, Liuzhi, Bijie, } \\
\text { Dafang, Guizhou }\end{array}$ & 3.9 & 71 & $\mathrm{P}_{2}$ & Dai et al. [94] & Tongchuan, Shaanxi & 2.43 & 2 & C-P & Ren et al. [39] \\
\hline Guiding, Guizhou & 9.24 & 14 & $\mathrm{P}_{2}$ & Dai et al. [98] & Weibei, Shaanxi & 7.595 & 48 & C-P & $\mathrm{Lu}[132]$ \\
\hline Zhenfeng Longtoushan, Guizhou & 54.77 & 7 & $\mathrm{P}_{3}$ & Zhang [63] & Hancheng and Tongchuan, Shaanxi & 3.88 & 14 & $\mathrm{C}-\mathrm{P}$ & Wang et al. [133] \\
\hline Puan, Guizhou & 10.18 & 5 & $\mathrm{P}_{2}$ & Yang [99] & Zichang, Shaanxi & 1.5 & 1 & $\mathrm{P}_{3}$ & Ren et al. [39] \\
\hline Changpo, Hainan & 8.07 & 1 & $\mathrm{E}-\mathrm{N}$ & Ren et al. [39] & Binxian, Shaanxi & 13.64 & 1 & $\mathrm{~J}_{1-2}$ & Li et al. [119] \\
\hline Tangshan Jinggezhuang, Hebei & 9.52 & 1 & $\mathrm{C}-\mathrm{P}$ & Zhuang et al. [100] & Junlian, Sichuan & 26.5 & 1 & $\mathrm{P}_{2}$ & Zhang et al. [107] \\
\hline Kailuan, Hebei & 6.14 & 47 & C-P & Tang et al. [101] & Guxu, Sichuan & 4.93 & 30 & $\mathrm{P}_{2}$ & Ren et al. [39] \\
\hline Kailuan, Hebei & 6.41 & 34 & $\mathrm{C}-\mathrm{P}$ & Zhuang et al. [100] & Junlian, Sichuan & 10.65 & 6 & $\mathrm{P}_{2}$ & Ren et al. [39] \\
\hline Fengfeng, Hebei & 2.38 & 9 & $\mathrm{C}-\mathrm{P}$ & Dai [102] & Furong, Sichuan & 9.48 & 5 & $\mathrm{P}_{2}$ & Ren et al. [39] \\
\hline Handan, Hebei & 3.83 & 3 & C-P & Ren et al. [39] & Dabaoding, Sichuan & 1.44 & 31 & $\mathrm{P}_{2}$ & this study \\
\hline Xingtai, Hebei & 1.6 & 3 & $\mathrm{C}-\mathrm{P}$ & Ren et al. [39] & Huayingshan, Sichuan & 3.15 & 5 & $\mathrm{P}_{2}$ & Dai et al. [134] \\
\hline JingXing and Yuanshi, Hebei & 2.28 & 2 & $\mathrm{C}-\mathrm{P}$ & Ren et al. [39] & Dukou, Sichuan & 1.99 & 2 & $\mathrm{P}_{3}$ & Ren et al. [39] \\
\hline Xinglong, Hebei & 3.53 & 1 & $\mathrm{C}-\mathrm{P}$ & Ren et al. [39] & Yaxing, Sichuan & 3.27 & 5 & $\mathrm{P}_{3}$ & Ren et al. [39] \\
\hline Fengfeng, Hebei & 15.03 & 15 & $\mathrm{P}_{2}$ & Dai and Ren [103] & Guxu, Sichuan & 2.252 & 11 & $\mathrm{P}_{2}$ & Dai et al. [135] \\
\hline Jiaozuo, Henan & 1.02 & 2 & $\mathrm{C}-\mathrm{P}$ & Ren et al. [39] & Taiwan & 25.38 & 4 & $\mathrm{E}-\mathrm{N}$ & Ren et al. [39] \\
\hline Pingdingshan, Henan & 1.93 & 22 & $\mathrm{C}-\mathrm{P}$ & Chen and Tang [72] & Tibet & 20.11 & 24 & $\mathrm{P}_{3}$ & Fu et al. [136] \\
\hline Hebi, Henan & 1.56 & 2 & $\mathrm{C}-\mathrm{P}$ & Zhao [30] & Kuche, Xinjiang & 0.85 & 15 & $\mathrm{~J}_{1-2}$ & Chen and Tang [72] \\
\hline Xinmi, Henan & 1.13 & 1 & $\mathrm{C}-\mathrm{P}$ & Ren et al. [39] & Yining, Xinjiang & 1.85 & 10 & $\mathrm{~J}_{1-2}$ & Chen and Tang [72] \\
\hline Yongcheng, Henan & 2 & 1 & $\mathrm{C}-\mathrm{P}$ & Ren et al. [39] & Santanghu, Xinjiang & 3.86 & 4 & $\mathrm{~J}_{1-2}$ & Chen and Tang [72] \\
\hline Yima, Henan & 19.44 & 5 & $\mathrm{~J}_{1-2}$ & Ren et al. [39] & Liuhuanggou, Xinjiang & 1.49 & 5 & $J_{1-2}$ & Chen and Tang [72] \\
\hline Jiayin, Heilongjiang & 2.77 & 1 & $\mathrm{E}-\mathrm{N}$ & Ren et al. [39] & Fukang, Xinjiang & 4.89 & 5 & $\mathrm{~J}_{1-2}$ & Chen and Tang [72] \\
\hline Jixi, Heilongjiang & 1.95 & 7 & $\mathrm{~J}_{3}-\mathrm{K}_{1}$ & Ren et al. [39] & Lucaogou, Xinjiang & 5.55 & 1 & $\mathrm{~J}_{1-2}$ & Ren et al. [39] \\
\hline Shuangyashan, Heilongjiang & 17.2 & 6 & $\mathrm{~J}_{3}-\mathrm{K}_{1}$ & Ren et al. [39] & Aiweiergou, Xinjiang & 0.96 & 10 & $J_{1-2}$ & Chen and Tang [72] \\
\hline Qitaihe, Heilongjiang & 1.85 & 3 & $\mathrm{~J}_{3}-\mathrm{K}_{1}$ & Ren et al. [39] & Hami, Xinjiang & 1.98 & 13 & $\mathrm{~J}_{1-2}$ & Chen and Tang [72] \\
\hline Suibin, Heilongjiang & 8.75 & 4 & $\mathrm{~J}_{3}-\mathrm{K}_{1}$ & Ren et al. [39] & Miquan, Xinjiang & 10.25 & 1 & $J_{1-2}$ & Ren et al. [39] \\
\hline Jixian, Heilongjiang & 4.47 & 8 & $\mathrm{~J}_{3}-\mathrm{K}_{1}$ & Ren et al. [39] & Dapugou, Xinjiang & 4.69 & 1 & $J_{1-2}$ & Ren et al. [39] \\
\hline Hegang, Heilongjiang & 1.67 & 1 & $\mathrm{~J}_{3}-\mathrm{K}_{1}$ & Ren et al. [39] & Yili, Xinjiang & 20.027 & 40 & $\mathrm{~J}_{1-2}$ & Dai et al. [137] \\
\hline Heihe, Heilongjiang & 2.85 & 1 & $\mathrm{~J}_{3}-\mathrm{K}_{1}$ & Ren et al. [39] & Hami, Xinjiang & 2.13 & 10 & $J_{1-2}$ & Tang et al. [66] \\
\hline Dazhi, Hubei & 17.26 & 3 & $\mathrm{P}_{2}$ & Ren et al. [39] & Fukang, Xinjiang & 1.24 & 4 & $\mathrm{~J}_{1-2}$ & Tang et al. [66] \\
\hline Songyi, Hubei & 4.58 & 1 & $\mathrm{P}_{2}$ & Ren et al. [39] & Aiweiergou, Xinjiang & 0.58 & 5 & $J_{1-2}$ & Tang et al. [66] \\
\hline Lichuan, Hubei & 8.3 & 1 & $\mathrm{P}_{2}$ & Ren et al. [39] & Kuche, Xinjiang & 0.59 & 6 & $\mathrm{~J}_{1-2}$ & Tang et al. [66] \\
\hline Hubei & 5.1 & 19 & & Cui and Chen [80] & Hetian, Xinjiang & 1.76 & 1 & $\mathrm{~J}_{1-2}$ & Tang et al. [66] \\
\hline Lianshao Jinzhushan, Hunan & 2 & 1 & $\mathrm{C}-\mathrm{P}$ & Ren et al. [39] & Zhunnan, Xinjiang & 1.49 & 5 & $J_{1-2}$ & Tang et al. [66] \\
\hline Lianshao Lengshuijiang, Hunan & 23 & 1 & $\mathrm{C}-\mathrm{P}$ & Ren et al. [39] & Kuche, Xinjiang & 2.18 & 9 & $\mathrm{~J}_{1-2}$ & Tang et al. [66] \\
\hline Zhadu, Hunan & 4.14 & 17 & $\mathrm{C}-\mathrm{P}$ & Yuan [104] & Lincang bangmai, Yunnan & 10.8 & 1 & $\mathrm{E}-\mathrm{N}$ & Tang et al. [66] \\
\hline Meitian, Hunan & 10 & 10 & $\mathrm{P}_{2}$ & Tang et al. [66] & Lincang, Yunnan & 12 & 4 & $\mathrm{E}-\mathrm{N}$ & Tang et al. [66] \\
\hline Lianshao, Hunan & 2.4 & 3 & $\mathrm{P}_{2}$ & Ren et al. [39] & Zhaotong, Yunnan & 9.75 & 25 & $\mathrm{E}-\mathrm{N}$ & Ren et al. [39] \\
\hline Baisha, Hunan & 4.75 & 1 & $\mathrm{P}_{2}$ & Ren et al. [39] & Xiaolongtan, Yunnan & 19.92 & 8 & $\mathrm{E}-\mathrm{N}$ & Ren et al. [39] \\
\hline Meitanba, Hunan & 6.05 & 1 & $\mathrm{P}_{2}$ & Ren et al. [39] & Xianfeng, Yunnan & 2.88 & 16 & $\mathrm{E}-\mathrm{N}$ & Ren et al. [39] \\
\hline Zixing, Hunan & 23.29 & 2 & $\mathrm{P}_{3}$ & Ren et al. [39] & Jinsuo, Yunnan & 7.01 & 2 & $\mathrm{E}-\mathrm{N}$ & $\mathrm{Gu}$ [138] \\
\hline
\end{tabular}


Table 1. Cont.

\begin{tabular}{|c|c|c|c|c|c|c|c|c|c|}
\hline Province/Coal Mine & $\begin{array}{l}\text { Arithmetic } \\
\text { Mean }\end{array}$ & $\begin{array}{c}\text { Sample } \\
\text { Number }\end{array}$ & $\begin{array}{c}\text { Coal-Forming } \\
\text { Period }\end{array}$ & Data Source & Province/Coal Mine & $\begin{array}{l}\text { Arithmetic } \\
\text { Mean }\end{array}$ & $\begin{array}{c}\text { Sample } \\
\text { Number }\end{array}$ & $\begin{array}{c}\text { Coal-Forming } \\
\text { Period }\end{array}$ & Data Source \\
\hline Meihe, Jilin & 6 & 1 & $\mathrm{E}-\mathrm{N}$ & Ren et al. [39] & Kebao, Yunnan & 5.09 & 19 & E-N & Ren et al. [39] \\
\hline Shulan, Jilin & 2.51 & 3 & $\mathrm{E}-\mathrm{N}$ & Ren et al. [39] & Fengmingcun, Yunnan & 2.5 & 1 & $\mathrm{E}-\mathrm{N}$ & Ren et al. [39] \\
\hline Baishan, Jilin & 7.45 & 56 & $\mathrm{C}-\mathrm{P}$ & Wu et al. [105] & Longling Daba, Yunnan & 6.98 & 2 & E-N & Ren et al. [39] \\
\hline Liaoyuan, Jilin & 21 & 1 & $\mathrm{~J}_{1-2}$ & Ren et al. [39] & Chuxiong, Yunnan & 17.39 & 3 & E-N & Ren et al. [39] \\
\hline Xuzhou Chacheng, Jiangsu & 1.1 & 7 & $\mathrm{C}-\mathrm{P}$ & Tang et al. [66] & Yaoan, Yunnan & 6.43 & 184 & $\mathrm{E}-\mathrm{N}$ & Ren et al. [39] \\
\hline Xuzhou, Jiangsu & 2.04 & 12 & $\mathrm{C}-\mathrm{P}$ & Chen and Tang [72] & Kunming, Yunnan & 25.8 & 23 & $\mathrm{E}-\mathrm{N}$ & Ren et al. [39] \\
\hline Fengpei, Jiangsu & 3.88 & 5 & C-P & Ren et al. [39] & Yuxi, Yunnan & 30.5 & 14 & E-N & Zhou [139] \\
\hline Zhenjiang, Jiangsu & 1.5 & 2 & $\mathrm{P}_{2}$ & Ren et al. [39] & Zhaotong, Yunnan & 12.7 & 24 & E-N & Zhou [139] \\
\hline Ganzhong, Jiangxi & 11.87 & 240 & $\mathrm{P}_{2}$ & Zhou [106] & Wandian, Yunnan & 21.4 & 33 & E-N & Zhou [139] \\
\hline Leping, Jiangxi & 9.5 & 1 & $\mathrm{P}_{2}$ & Zhang et al. [107] & Baolang, Yunnan & 7 & 17 & E-N & Zhou [139] \\
\hline Fengcheng, Jiangxi & 9.5 & 70 & $\mathrm{P}_{2}$ & Zhou [106] & Yaoan, Yunnan & 8.5 & 126 & E-N & Zhou [139] \\
\hline Yinggangling, Jiangxi & 10.9 & 192 & $\mathrm{P}_{2}$ & Zhou [106] & Lincang, Yunnan & 47.6 & 52 & E-N & Dai et al. [40] \\
\hline Yangqiao, Jiangxi & 4.18 & 21 & $\mathrm{P}_{2}$ & Zhou [106] & Lincang, Yunnan & 117 & 11 & E-N & Dai et al. [62] \\
\hline Pingxiang, Jiangxi & 1.65 & 8 & $\mathrm{P}_{3}$ & Ren et al. [39] & Yanshan, Yunnan & 9.1 & 3 & $\mathrm{P}_{2}$ & Dai et al. [140] \\
\hline Luoshi, Jiangxi & 3.1 & 70 & $\mathrm{P}_{3}$ & Zhou [106] & Laochang, Yunnan & 5 & 175 & $\mathrm{P}_{2}$ & Zhou [31] \\
\hline Huagushan, Jiangxi & 3.28 & 8 & $\mathrm{P}_{3}$ & Zhou [106] & Enhong, Yunnan & 1.77 & 55 & $\mathrm{P}_{2}$ & Zhou [31] \\
\hline Yongshan, Jiangxi & 11 & 7 & $\mathrm{P}_{3}$ & Ren et al. [39] & Yangchang, Yunnan & 0.9 & 97 & $\mathrm{P}_{2}$ & Zhou [31] \\
\hline Ganzhong, Jiangxi & 3.12 & 78 & $\mathrm{P}_{3}$ & Zhou [106] & Laibin, Yunnan & 1.29 & 12 & $\mathrm{P}_{2}$ & Zhou [31] \\
\hline Shenbei, Liaoning & 9.88 & 7 & $\mathrm{E}-\mathrm{N}$ & Ren et al. [108] & Housuo and Qingyun, Yunnan & 0.63 & 2 & $\mathrm{P}_{2}$ & Ren et al. [39] \\
\hline Fushunxi, Liaoning & 2.18 & 1 & $\mathrm{E}-\mathrm{N}$ & Tang et al. [66] & Qujing, Yunnan & 1.13 & 1 & $\mathrm{P}_{2}$ & Ren et al. [39] \\
\hline Fushun, Liaoning & 3.36 & 2 & $\mathrm{E}-\mathrm{N}$ & Ren et al. [39] & Luoping, Yunnan & 4.88 & 1 & $\mathrm{P}_{2}$ & Ren et al. [39] \\
\hline Shenbei, Liaoning & 16.85 & 12 & $\mathrm{E}-\mathrm{N}$ & Ren et al. [39] & Yanshan, Yunnan & 15.5 & 1 & $\mathrm{P}_{2}$ & Ren et al. [39] \\
\hline Tiefa, Liaoning & 4.1 & 3 & $\mathrm{~J}_{3}-\mathrm{K}_{1}$ & Ren et al. [39] & Xuanwei, Yunnan & 8.37 & 6 & $\mathrm{P}_{2}$ & Dai et al. [141] \\
\hline Fuxin, Liaoning & 4.39 & 7 & $\mathrm{~J}_{3}-\mathrm{K}_{1}$ & Zhuang et al. [100] & Yanshan, Yunnan & 9.14 & 6 & $\mathrm{P}_{2}$ & Dai et al. [142] \\
\hline Badaohao, Liaoning & 23.3 & 1 & $\mathrm{~J}_{3}-\mathrm{K}_{1}$ & Ren et al. [39] & Yipinglang, Yunnan & 21.85 & 2 & $\mathrm{P}_{3}$ & Ren et al. [39] \\
\hline Fuxin Haizhou, Liaoning & 4.98 & 6 & $\mathrm{~J}_{3}-\mathrm{K}_{1}$ & Querol et al. [109] & Chuxiong, Yunnan & 11.4 & 1 & $\mathrm{P}_{3}$ & Ren et al. [39] \\
\hline Beipiao, Liaoning & 3.73 & 1 & $\mathrm{~J}_{1-2}$ & Ren et al. [39] & Xinping, Yunnan & 4.6 & 1 & $\mathrm{P}_{3}$ & Ren et al. [39] \\
\hline Gongwusu, Inner Mongolia & 1.2 & 1 & $\mathrm{C}-\mathrm{P}$ & Wang et al. [110] & Xinde, Yunnan & 2.53 & 4 & $\mathrm{P}_{2}$ & Dai et al. [143] \\
\hline Wuda, Inner Mongolia & 1.57 & 4 & C-P & Dai [102] & Xinde, Yunnan & 1.14 & 3 & $\mathrm{P}_{2}$ & Dai et al. [143] \\
\hline Jungar, Inner Mongolia & 0.85 & 3 & C-P & $\operatorname{Li}[111]$ & Luquan, Yunnan & 5.28 & 8 & $\mathrm{D}$ & Dai et al. [144] \\
\hline Wuhai, Inner Mongolia & 3.96 & 1 & $\mathrm{C}-\mathrm{P}$ & Ren et al. [39] & Zhejiang & 11 & 5 & & Cui and Chen [80] \\
\hline Daqingshan, Inner Mongolia & 1.51 & 33 & $\mathrm{C}-\mathrm{P}$ & Dai et al. [112] & Changguang, Zhejiang & 13 & 8 & $\mathrm{P}_{2}$ & Ren et al. [39] \\
\hline
\end{tabular}


Table 2. Arsenic concentrations and predicted coal resources in individual provinces of China.

\begin{tabular}{|c|c|c|c|c|}
\hline Administrative Division & Predicted Resource/Billion Tons [64] & Sample Number & Arsenic Content Mean Value (mg/kg) & Concentration Coefficient \\
\hline Anhui & 611.59 & 350 & 6.32 & 1.67 \\
\hline Beijing & 86.72 & 4 & 1.47 & 0.39 \\
\hline Fujian & 25.57 & 5 & 10.20 & 2.69 \\
\hline Gansu & 1428.87 & 14 & 2.86 & 0.75 \\
\hline Guangdong & 9.11 & 35 & 9.68 & 2.55 \\
\hline Guangxi & 17.64 & 73 & 8.82 & 2.33 \\
\hline Guizhou & 1896.9 & 547 & 7.25 & 1.91 \\
\hline Hainan & 0.01 & 1 & 8.07 & 2.13 \\
\hline Hebei & 601.39 & 115 & 6.85 & 1.81 \\
\hline Henan & 919.71 & 33 & 4.48 & 1.18 \\
\hline Heilongjiang & 176.13 & 31 & 6.47 & 1.71 \\
\hline Hubei & 2.04 & 24 & 6.73 & 1.78 \\
\hline Hunan & 45.35 & 36 & 7.22 & 1.91 \\
\hline Jilin & 30.03 & 61 & 7.41 & 1.96 \\
\hline Jiangsu & 50.49 & 26 & 2.10 & 0.55 \\
\hline Jiangxi & 40.84 & 695 & 9.04 & 2.39 \\
\hline Liaoning & 59.27 & 40 & 9.51 & 2.51 \\
\hline Inner Mongolia & $12,250.4$ & 1053 & 3.80 & 1.00 \\
\hline Ningxia & 1721.11 & 30 & 1.72 & 0.45 \\
\hline Qinghai & 380.42 & 20 & 1.17 & 0.31 \\
\hline Shandong & 405.13 & 250 & 7.49 & 1.98 \\
\hline Shanxi & 3899.18 & 404 & 3.13 & 0.83 \\
\hline Shaanxi & 2031.1 & 69 & 6.43 & 1.70 \\
\hline Sichuan and Chongqing & 303.79 & 269 & 5.38 & 1.42 \\
\hline Taiwan & 1.8 & 4 & 25.38 & 6.70 \\
\hline Tibet & 8.09 & 24 & 20.11 & 5.31 \\
\hline Xinjiang & $18,037.3$ & 145 & 6.88 & 1.82 \\
\hline Yunnan & 437.87 & 943 & 10.82 & 2.85 \\
\hline Zhejiang & 0.44 & 13 & 12.23 & 3.23 \\
\hline \multirow[t]{2}{*}{ China } & $45,478.3$ & 5314 & 6.97 & \\
\hline & & & 5.33 (weighted average) & \\
\hline
\end{tabular}


Table 3. Arsenic content in Chinese coals (mg/kg). Data are quoted from references of $[3,19,21,23,39-41,44,45,47,56,57,66,145]$.

\begin{tabular}{|c|c|c|c|c|c|}
\hline Country & Arithmetic Mean & Geometric Mean & Resource Weighted Average & Sample Number & Data Source \\
\hline Chinese Coal & 276.61 & 4.26 & & 132 & Ren et al. [56] \\
\hline Chinese Coal & 4.7 & & & 1018 & Wang [145] \\
\hline Chinese Coal & 4 & & & 1915 & Chen et al. [57] \\
\hline Chinese Coal & 5 & & & 3193 & Tang et al. [66] \\
\hline Chinese Coal & 6.4 & 3.96 & & 297 & Wang [23] \\
\hline Chinese Coal & & & 3.79 & 3386 & Dai et al. [40] \\
\hline Chinese Coal & & & 3.80 & 3453 & Ren et al. [39] \\
\hline Chinese Coal & 9.70 & & 3.18 & 4805 & Kang [3] \\
\hline Chinese Coal & 6.97 & & 5.33 & 5314 & this study \\
\hline American Coal & 24 & 6.5 & & 7676 & Finkelman [41] \\
\hline American Coal & 24 & & & 6878 & Kolker et al. [21] \\
\hline Australian Coal & 2 & & & & Swaine and Goodarzi [44] \\
\hline Czech Coal & 39.94 & & & 9172 & Bouska and Pesek [45] \\
\hline World lignite & $7.6 \pm 1.3$ & & & 21,092 & Ketris and Yudovich [19] \\
\hline World bitumite & $9.0 \pm 0.7$ & & & 22,466 & Ketris and Yudovich [19] \\
\hline World Coal & 5 & & & & PECH [47] \\
\hline
\end{tabular}




\section{Distribution of Arsenic in Chinese Coals}

\subsection{Spatial Distribution Characteristics of Arsenic in Chinese Coals}

Based on the collected data of arsenic content in Chinese coals, the average arsenic contents in coals from different coal-bearing areas are calculated. Additionally, compared to average value of Chinese coals reported by Dai et al. [40], the concentration coefficient (CC) is given in Table 4 . When CC is above 100, it is abnormally enriched; when CC is above 10 but below 100, it is highly enriched; when CC is above 5 but below 10, it is enriched; when CC is above 2 but below 5 , it is slightly enriched; when CC is above 0.5 but below 2, it is normal; and when CC is below 0.5 , it is depleted [112].

In view of the concentration coefficient, the spatial variation of arsenic in Chinese coals can be classified as follow:

(1) Arsenic in coal is highly enriched in the southwestern Yunnan and part of Guizhou.

(2) Arsenic is enriched in the coals from some regions, such as the western Yunnan, Guangxi, Taiwan, Tibet, southwestern Liaoning, Jilin, and Henan.

(3) Arsenic in coal is slightly enriched in the southwestern and middle part of Guizhou, most of Yunnan, Guangdong, Guangxi, Hainan, Fujian, Jiangxi, Zhejiang, southeastern Hubei, southern Hunan, northwestern Chongqing, southwestern Shandong, southern Hebei, southern Shanxi, as well as parts of northern and southern Liaoning.

(4) Arsenic in coal is depleted in the western Xinjiang and most of Qinghai.

Overall, the content of arsenic in Chinese coals has an increasing tendency from the north to the south. Meanwhile, the content of arsenic in coal within coal-bearing basins differs spatially, due to factors, such as palaeomire conditions and provenance supply. The arsenic contents in coals from different provinces are various obviously. The arsenic content in coal has a significant correlation with coal-accumulation area. The distribution of arsenic in nationwide China is shown in Figure 1. The population of coal samples from Taiwan, Beijng, and Fujian are small and cannot well-represented local coals. 
Table 4. Spatial variation of arsenic in Chinese coals.

\begin{tabular}{|c|c|c|c|c|c|c|c|c|}
\hline Province/Area & Anhui Province & $\begin{array}{l}\text { Northern } \\
\text { Anhui }\end{array}$ & $\begin{array}{l}\text { Southern } \\
\text { Anhui }\end{array}$ & $\begin{array}{l}\text { North Central } \\
\text { of Anhui }\end{array}$ & Beijing & $\begin{array}{l}\text { Western } \\
\text { Beijing }\end{array}$ & Fujian Province & Central of Fujian \\
\hline Mean Value & 6.32 & 2.35 & 10.16 & 6.37 & 1.47 & 1.00 & 10.20 & 12.00 \\
\hline CC & 1.67 & 0.62 & 2.68 & 1.68 & 0.39 & 0.26 & 2.69 & 3.17 \\
\hline Province/Area & Gansu Province & Eastern Gansu & $\begin{array}{l}\text { Southeastern } \\
\text { Gansu }\end{array}$ & $\begin{array}{l}\text { Northwestern } \\
\text { Gansu }\end{array}$ & $\begin{array}{c}\text { Central of } \\
\text { Gansu }\end{array}$ & $\begin{array}{l}\text { Guangdong } \\
\text { Province }\end{array}$ & $\begin{array}{l}\text { Northeastern } \\
\text { Guangdong }\end{array}$ & $\begin{array}{l}\text { Southern } \\
\text { Guangdong }\end{array}$ \\
\hline Mean Value & 2.86 & 3.33 & 1.50 & 3.06 & 2.99 & 9.68 & 8.00 & 13.90 \\
\hline $\mathrm{CC}$ & 0.75 & 0.88 & 0.40 & 0.81 & 0.79 & 2.55 & 2.11 & 3.67 \\
\hline Province/Area & Guangxi & $\begin{array}{l}\text { Northern } \\
\text { Guangxi }\end{array}$ & $\begin{array}{l}\text { Southern } \\
\text { Guangxi }\end{array}$ & $\begin{array}{l}\text { Western } \\
\text { Guangxi }\end{array}$ & $\begin{array}{l}\text { Southwestern } \\
\text { Guangxi }\end{array}$ & $\begin{array}{l}\text { Central of } \\
\text { Guangxi }\end{array}$ & $\begin{array}{l}\text { Guizhou } \\
\text { Province }\end{array}$ & Northern Guizhou \\
\hline Mean Value & 8.82 & 3.84 & 19.60 & 25.40 & 8.59 & 7.81 & 7.25 & 3.19 \\
\hline CC & 2.33 & 1.01 & 5.17 & 6.70 & 2.27 & 2.06 & 1.91 & 0.84 \\
\hline Province/Area & $\begin{array}{l}\text { Northwestern } \\
\text { Guizhou }\end{array}$ & $\begin{array}{l}\text { Western } \\
\text { Guizhou }\end{array}$ & $\begin{array}{l}\text { Southwestern } \\
\text { Guizhou }\end{array}$ & $\begin{array}{l}\text { Central of } \\
\text { Guizhou }\end{array}$ & $\begin{array}{l}\text { Hainan } \\
\text { Province }\end{array}$ & $\begin{array}{l}\text { Northwestern } \\
\text { Hainan }\end{array}$ & Hebei Province & Northeastern Hebei \\
\hline Mean Value & 5.78 & 5.91 & 12.41 & 8.01 & 8.07 & 8.07 & 6.85 & 3.53 \\
\hline $\mathrm{CC}$ & 1.52 & 1.56 & 3.28 & 2.11 & 2.13 & 2.13 & 1.81 & 0.93 \\
\hline Province/Area & Eastern Hebei & Southern Hebei & Western Hebei & Henan Province & Northern Henan & Eastern Henan & Western Henan & Central of Henan \\
\hline Mean Value & 6.29 & 8.77 & 2.28 & 4.48 & 1.29 & 2.00 & 19.44 & 1.90 \\
\hline $\mathrm{CC}$ & 1.66 & 2.31 & 0.60 & 1.18 & 0.34 & 0.53 & 5.13 & 0.50 \\
\hline Province/Area & $\begin{array}{l}\text { Heilongjiang } \\
\text { Province }\end{array}$ & $\begin{array}{c}\text { Northern } \\
\text { Heilongjiang }\end{array}$ & $\begin{array}{l}\text { Northeastern } \\
\text { Heilongjiang }\end{array}$ & $\begin{array}{c}\text { Eastern } \\
\text { Heilongijiang }\end{array}$ & $\begin{array}{l}\text { Southeastern } \\
\text { Heilongjiang }\end{array}$ & $\begin{array}{c}\text { Northwestern } \\
\text { Heilongjiang }\end{array}$ & Hubei Province & Southeastern Hubei \\
\hline Mean Value & 6.47 & 2.77 & 9.24 & 1.85 & 1.95 & 2.85 & 6.73 & 17.26 \\
\hline $\mathrm{CC}$ & 1.71 & 0.73 & 2.44 & 0.49 & 0.51 & 0.75 & 1.78 & 4.55 \\
\hline Province/Area & $\begin{array}{c}\text { Southwestern } \\
\text { Hubei }\end{array}$ & Hunan Province & $\begin{array}{l}\text { Southern } \\
\text { Hunan }\end{array}$ & $\begin{array}{l}\text { Central of } \\
\text { Hunan }\end{array}$ & Jilin Province & Northern Jilin & Southern Jilin & $\begin{array}{l}\text { South Central of } \\
\text { Jilin }\end{array}$ \\
\hline Mean Value & 6.44 & 7.22 & 11.64 & 4.72 & 7.41 & 2.51 & 7.42 & 21.00 \\
\hline $\mathrm{CC}$ & 1.70 & 1.91 & 3.07 & 1.25 & 1.95 & 0.66 & 1.96 & 5.54 \\
\hline Province/Area & $\begin{array}{l}\text { Jiangsu } \\
\text { Province }\end{array}$ & $\begin{array}{c}\text { Northwestern } \\
\text { Jiangsu }\end{array}$ & $\begin{array}{l}\text { Southwestern } \\
\text { Jiangsu }\end{array}$ & Jiangxi Province & $\begin{array}{c}\text { Northeastern } \\
\text { Jiangxi }\end{array}$ & Western Jiangxi & $\begin{array}{c}\text { Central of } \\
\text { Jiangxi }\end{array}$ & Liaoning Province \\
\hline Mean Value & 2.10 & 2.15 & 1.50 & 9.04 & 10.81 & 8.15 & 9.68 & 9.51 \\
\hline CC & 0.55 & 0.57 & 0.40 & 2.38 & 2.85 & 2.15 & 2.55 & 2.51 \\
\hline Province/Area & $\begin{array}{l}\text { Northern } \\
\text { Liaoning }\end{array}$ & $\begin{array}{l}\text { Eastern } \\
\text { Liaoning }\end{array}$ & $\begin{array}{l}\text { Western } \\
\text { Liaoning }\end{array}$ & $\begin{array}{l}\text { Southwestern } \\
\text { Liaoning }\end{array}$ & $\begin{array}{c}\text { Inner } \\
\text { Mongolia(IM) }\end{array}$ & $\begin{array}{c}\text { Northeastern } \\
\text { IM }\end{array}$ & Southeastern IM & Western IM \\
\hline
\end{tabular}


Table 4. Cont

\begin{tabular}{|c|c|c|c|c|c|c|c|c|}
\hline Province/Area & Anhui Province & $\begin{array}{l}\text { Northern } \\
\text { Anhui }\end{array}$ & $\begin{array}{l}\text { Southern } \\
\text { Anhui }\end{array}$ & $\begin{array}{c}\text { North Central } \\
\text { of Anhui }\end{array}$ & Beijing & $\begin{array}{l}\text { Western } \\
\text { Beijing }\end{array}$ & Fujian Province & Central of Fujian \\
\hline Mean Value & 12.89 & 2.97 & 4.60 & 23.30 & 3.80 & 7.83 & 2.95 & 2.59 \\
\hline $\mathrm{CC}$ & 3.40 & 0.78 & 1.21 & 6.15 & 1.00 & 2.07 & 0.78 & 0.68 \\
\hline Province/Area & $\begin{array}{c}\text { Southwestern } \\
\text { IM }\end{array}$ & Midwestern IM & Ningxia & $\begin{array}{l}\text { Northern } \\
\text { Ningxia }\end{array}$ & $\begin{array}{l}\text { Central of } \\
\text { Ningxia }\end{array}$ & $\begin{array}{l}\text { Qinghai } \\
\text { Province }\end{array}$ & $\begin{array}{l}\text { Northern } \\
\text { Qinghai }\end{array}$ & Eastern Qinghai \\
\hline Mean Value & 3.76 & 1.39 & 1.72 & 1.12 & 4.13 & 1.17 & 0.90 & 3.34 \\
\hline CC & 0.99 & 0.37 & 0.45 & 0.30 & 1.09 & 0.31 & 0.24 & 0.88 \\
\hline Province/Area & $\begin{array}{l}\text { Western } \\
\text { Qinghai }\end{array}$ & $\begin{array}{l}\text { Shandong } \\
\text { Province }\end{array}$ & $\begin{array}{l}\text { Northeastern } \\
\text { Shandong }\end{array}$ & $\begin{array}{l}\text { Southern } \\
\text { Shandong }\end{array}$ & $\begin{array}{l}\text { Western } \\
\text { Shandong }\end{array}$ & $\begin{array}{l}\text { Southwestern } \\
\text { Shandong }\end{array}$ & $\begin{array}{l}\text { Central of } \\
\text { Shandong }\end{array}$ & Shanxi Province \\
\hline Mean Value & 1.34 & 7.49 & 2.90 & 5.23 & 7.90 & 8.78 & 6.12 & 3.13 \\
\hline $\mathrm{CC}$ & 0.35 & 1.98 & 0.77 & 1.38 & 2.08 & 2.32 & 1.61 & 0.83 \\
\hline Province/Area & $\begin{array}{l}\text { Northern } \\
\text { Shanxi }\end{array}$ & $\begin{array}{l}\text { Northeastern } \\
\text { Shanxi }\end{array}$ & Eastern Shanxi & $\begin{array}{c}\text { Southeastern } \\
\text { Shanxi }\end{array}$ & Southern Shanxi & $\begin{array}{c}\text { Northwestern } \\
\text { Shanxi }\end{array}$ & $\begin{array}{c}\text { Southwestern } \\
\text { Shanxi }\end{array}$ & Central of Shanxi \\
\hline Mean Value & 7.89 & 10.57 & 1.14 & 2.01 & 7.70 & 1.47 & 1.31 & 3.49 \\
\hline $\mathrm{CC}$ & 2.08 & 2.79 & 0.30 & 0.53 & 2.03 & 0.39 & 0.35 & 0.92 \\
\hline Province/Area & $\begin{array}{l}\text { Shaanxi } \\
\text { Province }\end{array}$ & $\begin{array}{l}\text { Northern } \\
\text { Shaanxi }\end{array}$ & $\begin{array}{l}\text { Central of } \\
\text { Shaanxi }\end{array}$ & $\begin{array}{l}\text { Sichuan } \\
\text { Province }\end{array}$ & $\begin{array}{c}\text { Northeastern } \\
\text { Sichuan }\end{array}$ & Eastern Sichuan & $\begin{array}{c}\text { Southeastern } \\
\text { Sichuan }\end{array}$ & $\begin{array}{l}\text { Southwestern } \\
\text { Sichuan }\end{array}$ \\
\hline Mean Value & 6.43 & 1.50 & 6.50 & 4.07 & 1.99 & 3.15 & 5.86 & 1.44 \\
\hline $\mathrm{CC}$ & 1.70 & 0.40 & 1.71 & 1.07 & 0.53 & 0.83 & 1.55 & 0.38 \\
\hline Province/Area & $\begin{array}{l}\text { Central of } \\
\text { Sichuan }\end{array}$ & Taiwan & Tibet & Xinjiang & $\begin{array}{c}\text { Northern } \\
\text { Xinjiang }\end{array}$ & Eastern Xinjiang & $\begin{array}{l}\text { Northwestern } \\
\text { Xinjiang }\end{array}$ & $\begin{array}{c}\text { Southwestern } \\
\text { Xinjiang }\end{array}$ \\
\hline Mean Value & 3.27 & 25.38 & 20.11 & 6.88 & 2.63 & 2.31 & 16.39 & 1.76 \\
\hline $\mathrm{CC}$ & 0.86 & 6.70 & 5.31 & 1.81 & 0.69 & 0.61 & 4.32 & 0.46 \\
\hline Province/Area & $\begin{array}{l}\text { North Central of } \\
\text { Xinjiang }\end{array}$ & $\begin{array}{c}\text { Midwest of } \\
\text { Xinjiang }\end{array}$ & $\begin{array}{c}\text { Yunnan } \\
\text { Province }\end{array}$ & $\begin{array}{l}\text { Northeastern } \\
\text { Yunnan }\end{array}$ & Eastern Yunnan & $\begin{array}{c}\text { Southeastern } \\
\text { Yunnan }\end{array}$ & Western Yunnan & $\begin{array}{l}\text { Southwestern } \\
\text { Yunnan }\end{array}$ \\
\hline Mean Value & 1.76 & 1.20 & 10.82 & 9.03 & 3.13 & 14.28 & 20.58 & 56.19 \\
\hline $\mathrm{CC}$ & 0.46 & 0.32 & 2.85 & 2.38 & 0.83 & 3.77 & 5.43 & 14.83 \\
\hline Province/Area & $\begin{array}{l}\text { Central of } \\
\text { Yunnan }\end{array}$ & $\begin{array}{l}\text { Zhejiang } \\
\text { Province }\end{array}$ & $\begin{array}{l}\text { Northern } \\
\text { Zhejiang }\end{array}$ & Chongqing & $\begin{array}{l}\text { Northern } \\
\text { Chongqing }\end{array}$ & $\begin{array}{c}\text { Southeastern } \\
\text { Chongqing }\end{array}$ & $\begin{array}{l}\text { Southern } \\
\text { Chongqing }\end{array}$ & Western Chongqing \\
\hline Mean Value & 9.14 & 12.23 & 13.00 & 6.11 & 7.88 & 7.40 & 4.96 & 8.77 \\
\hline $\mathrm{CC}$ & 2.41 & 3.23 & 3.43 & 1.61 & 2.08 & 1.95 & 1.31 & 2.31 \\
\hline
\end{tabular}

Concentration coefficient short for CC. 


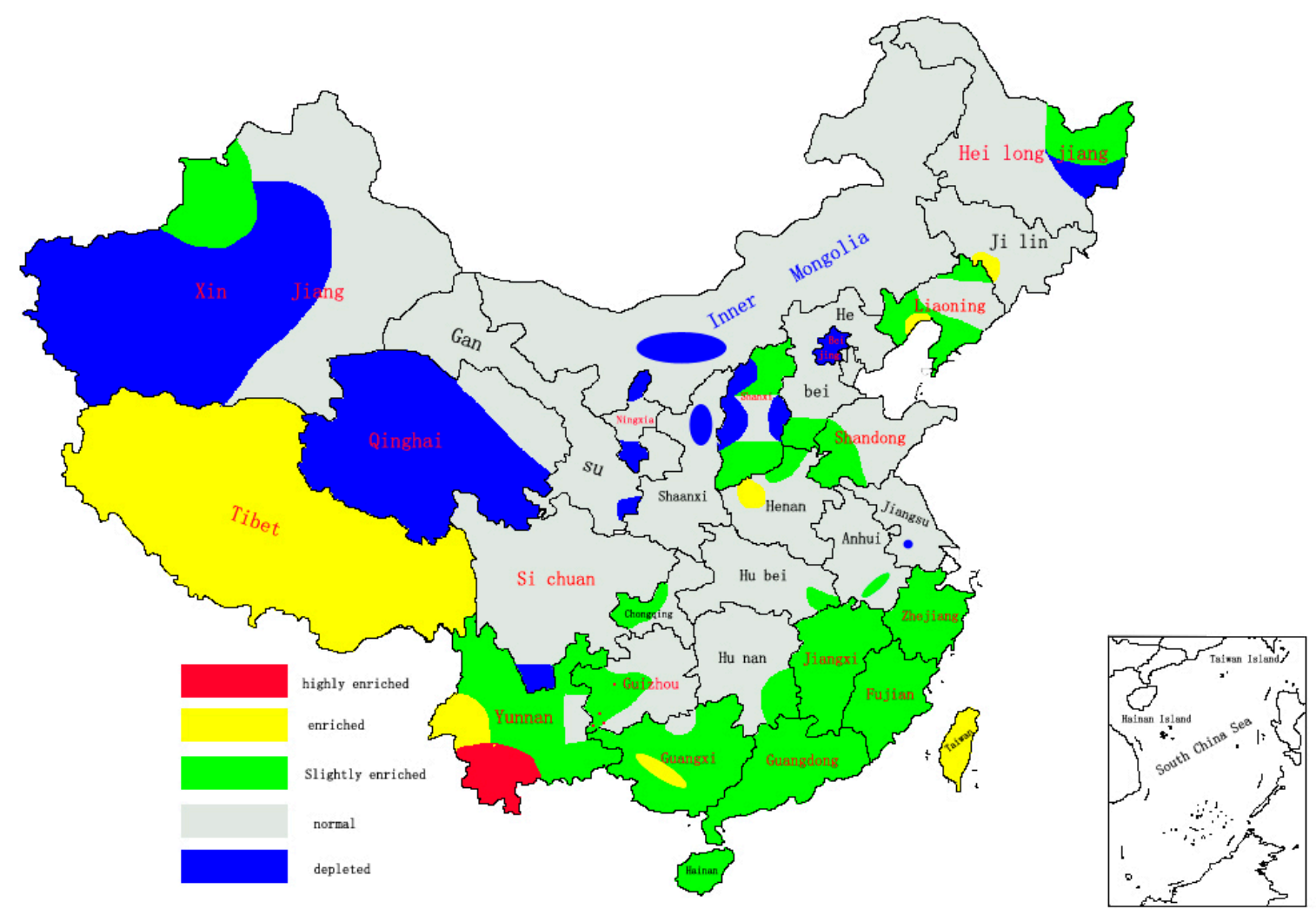

Figure 1. Arsenic distribution in Chinese coals by provinces.

\subsection{Distribution Characteristic of Arsenic in Chinese Coal in Different Coal-Forming Periods}

There are six major coal-forming periods in China: Late Carboniferous and Early Permian $\left(\mathrm{C}_{2}-\mathrm{P}_{1}\right)$, Late Permian $\left(\mathrm{P}_{2}\right)$, Late Triassic $\left(\mathrm{T}_{3}\right)$, Early and Middle Jurassic $\left(\mathrm{J}_{1-2}\right)$, Late Jurassic and Early Cretaceous $\left(\mathrm{J}_{3}-\mathrm{K}_{1}\right)$, and Paleogene and Neogene (E-N) [39]. Coals of these periods individually account for $38.1 \%$, $7.5 \%, 0.4 \%, 39.6 \%, 12.1 \%$, and $2.3 \%$ of the total Chinese reserves based on the Third National Prediction of Coal Resources of China [40].

The arsenic contents in coals of various coal-forming periods exhibit a significant difference [146]. The calculated contents of arsenic in coals shown in Table 5 exhibit the following regularity: Paleogene and Neogene $>$ Late Triassic > Late Permian > Late Jurassic and Early Cretaceous > Early and Middle Jurassic > Late Carboniferous and Early Permian, which is similar to the trend reported by Wang [23]. However, it differs from the distribution reported by Zheng et al. [146] and Lv et al. [147], who considered the averaging arsenic content in the Triassic coals was at first. The content of arsenic in low rank coals is highest in the Paleogene and Neogene, which is consistent with the results of Zhou [139], Li et al. [148], and Dai et al. [62]. Due to the great number of samples and an elaborative analysis and verification of the data sources, this statistical conclusion might be credible.

Table 5. Arsenic concentration in coals of different coal-forming periods in China $(\mathrm{mg} / \mathrm{kg})$.

\begin{tabular}{cccc}
\hline Coal-Forming Periods & $\begin{array}{c}\text { Arithmetic } \\
\text { Mean }\end{array}$ & $\begin{array}{c}\text { Sample } \\
\text { Number }\end{array}$ & $\begin{array}{c}\text { Recoverable Coal } \\
\text { Reserve (\%) }\end{array}$ \\
\hline Late Carboniferous and Early Permian & 4.63 & 1316 & 38.1 \\
Late Permian & 7.13 & 1839 & 7.5 \\
Late Triassic & 7.76 & 257 & 0.4 \\
Early and Middle Jurassic & 4.66 & 1141 & 39.6 \\
Late Jurassic and Early Cretaceous & 6.88 & 88 & 12.1 \\
Paleogene and Neogene & 15.5 & 606 & 2.3 \\
\hline
\end{tabular}




\subsection{Profile Distribution of Arsenic in Chinese Coals}

From a profile distribution perspective, there are obvious variations of arsenic content in different coal seams. Generally, arsenic variation in the coal-bearing profiles can be divided into several types: (1) Arsenic enriches in the roof, floor, coal seam and parting materials, e.g., the Xinlongchang and Jiaole coal mines in the Xingren of Guizhou Province [149]; (2) Arsenic enriches in the roof while depletes in the coal seam, e.g., the No. 15 coal of Qinshui Basin [150], the Nos. 4 and 6 coals of the Donglin coal mine in Nantong coalfield of Chongqing [151], and the No. 24 coal of the Taiping coal mine in the Panzhihua; (3) Arsenic enriches in the floor while depletes in the coal seam, e.g., the No. 3 coal of the Qinshui Basin [150] and the Xiashan coal mine in the Xingren of Guizhou Province [149]; (4) Arsenic in both the roof and floor are enriched, e.g., the Taiyang coal mine in the middle of Jiangxi Province [106]; And (5) arsenic content presents no obvious variation in one coal seam, e.g., the No. 5 coal of Chuancaogedan in Jungar Coalfield [114]. In a thick, multi-layer coal seam of the Panjiazhuang coal mine in the Xinren of Guizhou Province, only one layer contains high concentrations of As [149].

\section{Modes of Occurrence of Arsenic in Chinese Coals}

The modes of occurrence of arsenic in coals are of significance in the understanding of arsenic accumulation, migration mechanism, proper utilization of coal resources, and decrease of environment problems [23]. Arsenic in coals can be classified into the inorganic and organic arsenic. The relationship between minerals (such as pyrite, marcasite, and clay minerals) and arsenic in coals were widely investigated [30,117,152-154]. Although the detailed structure of organic arsenic in coal is still uncertain, many researchers $[28,32,152,155]$ have a positive view on the existence of organic arsenic. The modes of occurrence of arsenic in Chinese coals include sulfide-association, organic-association, arsenate-association, silicate-association, and soluble- and exchangeable-association.

\subsection{Sulfide-Association}

Arsenic in coal usually co-exists with pyrite $[28,152-154]$, but it is rare to find arsenic in the form of realgar and orpiment. Zhou [31] researched anthracite coal in the Late Permian Laochang mining area in the eastern Yunnan and discovered that the pyrite was the main carrier of arsenic when sulfur contents higher than $0.6 \%$. By sequential extraction, Guo et al. [156] measured the occurrence of arsenic in anthracite, lignite and bituminous coal, indicating that $73 \%-83 \%$ arsenic was bound to sulfide. Moreover, Zhao et al. [152] found that sulfide-bound arsenic, as pyrite, accounts for $0-85 \%$, with an average of $36 \%$. Arsenic in pyrite in coal exists mainly in the form of arsenic-bearing pyrite, rather than arsenopyrite. By electronic probe analysis on a high arsenic coal in the western Guizhou, Nie and Xie [157] confirmed that arsenic existed mainly in pyrite, and with distinctly different arsenic content on the high side of the secondary pyrite. The results of Zhao [30] and Zhang [63] on the arsenic in pyrite of the Late Permian in Guizhou suggested that the arsenic in epigenetic low-temperature hydrothermal vein pyrite was higher than that of syngenetic pyrite. The arsenic enrichment in Guizhou coals resulted from the epigenetic low-temperature hydrothermal fluids. Chen et al. [151] also found that arsenic primarily associated with pyrite in the Donglin coal from Nantong coalfield of Chongqing.

\subsection{Organic-Association}

Organic-associated arsenic is ubiquitous in coal and the proportion of organic-associated arsenic varies considerably among different coal samples. Using an extraction experiment of arsenic in low rank Xiaolongtan coals from the Yunnan Province, Zhang and Fan [155] discovered that more than $80 \%$ of total arsenic was organic associated. Wang et al. [158] found that organic-associated arsenic accounted for $51.38 \%-100 \%$ in the Jincheng coal from Shanxi Province. Zhao et al. [89] concluded that when the content of arsenic in coal was below $5.5 \mathrm{mg} / \mathrm{kg}$ and ash content was below $30 \%$, arsenic presented as organic-association. About $8 \%$ arsenic in the Laiyang anthracite coal, Qianjiaying lignite, and Qingshan bituminous coal was organic-associated [156]. Liu et al. [159] and Ding et al. [160] 
focused on the Yanzhou coal and the high-arsenic coal in the southwestern Guizhou, respectively, an organic-association arsenic was reported.

\subsection{Arsenate-Association}

Leaching experiments on lignites from Yunnan indicate that besides the arsenate absorbed by iron oxide and hydroxide, the arsenic mainly exists in limonite, magnetite, hematite, and other iron minerals [138]. Ren et al. [56] also found that arsenic occurred as arsenate in coals. Zhao et al. [59] and Ding et al. [32] investigated the Late Permian high-arsenic coal from Guizhou Province, arsenic were found in forms of arsenate or arsenite. Further, Zhao et al. [152] reported that arsenic combined with arsenate in coals accounts for approximately $0-65 \%$, with $17 \%$ on average. The proportion of arsenate-state arsenic is positively correlated with the iron content in coal [152].

\subsection{Silicate-Association}

Chen et al. [57] found that the arsenic presents an increasing trend with the increase of $\mathrm{Al}_{2} \mathrm{O}_{3}$ and $\mathrm{Fe}_{2} \mathrm{O}_{3}$. And they inferred that the clay minerals contain arsenic. By the sequential chemical extraction experiments on some samples from the Guizhou and Shanxi, Zhao et al. [152] found that silicate-combined arsenic accounts for approximately $0-90 \%$ of total arsenic, with an average of $16 \%$, which is proportional to the logarithm of ash yield in coal. The silicate-associated arsenic that is extracted from coal by hydrofluoric acid is mainly in the crystal lattice of clay minerals.

\subsection{Soluble- and Exchangeable-Association}

Arsenic in soluble and exchangeable forms is easily mobilized, and thus, have an adverse impact on the environment and human health. In a Huainan coal, Kang [3] reported that the ratio of the total soluble to exchangeable arsenic is between $1.78 \%$ and $6.28 \%$, illustrating that the arsenic in coal has certain dissolution ability and could be released into the surface environment by rainfall.

\subsection{Summary of Modes of Occurrence of Arsenic in Chinese Coals}

Based on the sequential chemical extraction experiments on high-arsenic coal in the Guizhou, Ding [161] found that organic-associated arsenic accounts for $0-80 \%$, silicate-associated arsenic accounts for $15 \%-90 \%$, sulfide-associated arsenic accounts for $0-25 \%$, and arsenate-associated arsenic accounts for 5\%-65\%. Kang [3] indicated that the modes of occurrence of arsenic in the Huainan coals are mainly sulfide-associated, partially in organic and silicate combined states. Zhao et al. [152] provided a sequence of the range of arsenic modes of occurrence in coals: sulfide-associated $(36 \%)>$ organic-associated $(26 \%)>$ arsenate-associated $(17 \%)>$ silicate-associated $(16 \%)>$ solubleand exchangeable-associated (5\%). The arsenic in Chinese coals mainly occurs in the form of sulfides-association with considerable differences among the coal samples. Arsenic in As-rich coal is often associated with minerals of epigenetic hydrothermal solution origins.

\section{Conclusions}

(1) Based on 5314 samples of Chinese coals, the arithmetic mean of the arsenic is calculated as $6.97 \mathrm{mg} / \mathrm{kg}$. Selecting the percentage of coal resources in each province of the total national resources as the weighting factor, the weighted arsenic average is $5.33 \mathrm{mg} / \mathrm{kg}$. Although the arsenic appears abnormally enriched in some Guizhou and Yunnan coals, the common Chinese coal is still comparable to the world level.

(2) The content of arsenic in Chinese coals increases from the north to the south. High-arsenic coal is mainly located in the southwestern Yunnan and part of Guizhou Province. Arsenic is enriched in the coals from some regions, i.e., the western Yunnan, Guangxi, Tibet, southwestern Liaoning, Jilin, and Henan. 
(3) The arsenic content in coals of different coal-forming periods shows an overall regularity: Paleogene and Neogene > Late Triassic $>$ Late Permian > Late Jurassic and Early Cretaceous > Early and Middle Jurassic > Late Carboniferous and Early Permian.

(4) The majority of arsenic in Chinese coals exists in arsenic-bearing pyrite. In coal samples with overall low arsenic content, the organic arsenic is dominant.

(5) The distribution and modes of occurrence of arsenic in Chinese coals are impacted by many factors, e.g., the coal-forming material, depositional environment, and epigenetic processes. Thus, the arsenic distribution is nonuniform, and the modes of occurrence exhibit a diversity and complexity. This needs further investigation and assessment by some advanced methods, such as the XAFS, MSXRF, and XANES spectrum.

Acknowledgments: This research was supported by National Basic Research Program of China (No. 2014CB238901). Special thanks are given to Shifeng Dai and three anonymous reviewers for their useful suggestions and comments.

Author Contributions: Duoxi Yao and Ping Chen applied for funding for this work. Duoxi Yao and Jiangfeng Guo designed this project. Jian Chen, Jiangfeng Guo and Fengjun Shi collected and analyzed the data. Jiangfeng Guo and Duoxi Yao prepared the first draft of this paper. Jian Chen and Ping Chen revised the paper and provided some contributions to the data interpretation.

Conflicts of Interest: The authors declare no conflict of interest.

\section{References}

1. BP Statistical Review of World Energy 2017. Available online: http://www.bp.com/en/global/corporate/ energy-economics/statistical-review-of-world-energy.html (accessed on 23 June 2017).

2. Statistical Communique on China's 2016 National Economic and Social Development. Available online: http://www.stats.gov.cn/tjsj/zxfb./201702/t20170228_1467424.html (accessed on 28 February 2017).

3. Kang, Y. Environmental Biogeochemistry of Arsenic in Coal Mining Area. Ph.D. Thesis, University of Science and Technology of China, Hefei, China, 2014. (In Chinese)

4. Zhang, H.J.; Yang, Y.Q.; Shi, X.; Mi, J. Impairment of Arsenic in Coal on Environment and Human Health. Sci.Tech. Inf. Dev. Econ. 2005, 15, 138-139. (In Chinese)

5. Sun, J.M.; Yao, Q.; Liu, H.Y.; Lu, J.; Yin, G.X.; Zhao, C.M. Distribution of arsenic in $\mathrm{PM}_{10} \& \mathrm{PM}_{2.5}$ caused by coal combustion and its enrichment mechanism. J. China Coal Soc. 2004, 29, 78-82. (In Chinese)

6. Nriagu, J.O.; Azcue, J.M. Environmental sources of arsenic in food. Adv. Environ. Sci. Technol. 1990, 23, 103-127.

7. Matschullat, J. Arsenic in the geosphere-A review. Sci. Total Environ. 2000, 249, 297-312. [CrossRef]

8. Tian, H.Z.; Qu, Y.P. Inventories of Atmospheric Arsenic Emissions from Coal Combustion in China, 2005. China Environ. Sci. 2009, 30, 956-962. (In Chinese)

9. Wei, Q.; Rimmer, S.M. Acid solubility and affinities of trace elements in the high-Ge coals from Wulantuga (Inner Mongolia) and Lincang (Yunnan Province), China. Int. J. Coal Geol. 2017, 178, 39-55. [CrossRef]

10. Dai, S.F.; Finkelman, R.B. Coal as a promising source of critical elements: Progress and future prospects. Int. J. Coal Geol. 2017, in press. [CrossRef]

11. Dai, S.F.; Yan, X.Y.; Ward, C.R.; Hower, J.C.; Zhao, L.; Wang, X.B.; Zhao, L.X.; Ren, D.Y.; Finkelman, R.B. Valuable elements in Chinese coals: A review. Int. Geol. Rev. 2016, 1-31. [CrossRef]

12. Dai, S.F.; Seredin, V.V.; Ward, C.R.; Jiang, J.H.; Hower, J.C.; Song, X.L.; Jiang, Y.F.; Wang, X.B.; Gornostaeva, T.; $\mathrm{Li}, \mathrm{X}$; et al. Composition and modes of occurrence of minerals and elements in coal combustion products derived from high-Ge coals. Int. J. Coal Geol. 2014, 121, 79-97. [CrossRef]

13. Swaine, D.J. Trace Elements in Coal; Butterworths: London, UK, 1990; pp. 1-83.

14. Dai, S.F.; Wang, X.B.; Zhou, Y.P.; Hower, J.C.; Li, D.H.; Chen, W.M.; Zhu, X.W.; Zou, J.H. Chemical and mineralogical compositions of silicic, mafic, and alkali tonsteins in the late Permian coals from the Songzao Coalfield, Chongqing, Southwest China. Chem. Geol. 2011, 282, 29-44. [CrossRef] 
15. Dai, S.F.; Wang, X.B.; Seredin, V.V.; Hower, J.C.; Ward, C.R.; O’Keefe, J.M.K.; Huang, W.H.; Li, T.; Li, X.; Liu, H.D.; et al. Petrology, mineralogy, and geochemistry of the Ge-rich coal from the Wulantuga Ge ore deposit, Inner Mongolia, China: New data and genetic implications. Int. J. Coal Geol. 2012, 90-91, 72-99. [CrossRef]

16. Li, X.; Dai, S.F.; Zhang, W.G.; Li, T.; Zheng, X.; Chen, W.M. Determination of As and Se in coal and coal combustion products using closed vessel microwave digestion and collision/reaction cell technology (CCT) of inductively coupled plasma mass spectrometry (ICP-MS). Int. J. Coal Geol. 2014, 124, 1-4. [CrossRef]

17. Valkovic, V.V. Trace Elements in Coal; CRC Press: Boca Raton, FL, USA, 1983.

18. Yudovich, Y.E.; Ketris, M.P.; Merts, A.V. Trace Elements in Fossil Coals; Nauka Science Publishing House: Leningrad, USSR, Russia, 1985.

19. Ketris, M.P.; Yudovich, Y.E. Estimations of Clarkes for Carbonaceous biolithes: World averages for trace element contents in black shales and coals. Int. J. Coal Geol. 2009, 78, 135-148. [CrossRef]

20. Finkelman, R.B. Modes of Occurrence of Trace Elements and Minerals in Coal: An Analytical Approach. In Atomic and Nuclear Methods in Fossil Energy Research; Filby, R.H., Carpenter, B.S., Ragaini, R.C., Eds.; Springer: New York, NY, USA, 1982; pp. 141-149.

21. Kolker, A.; Palmer, C.A.; Bragg, L.J.; Bunnell, J.E. Arsenic in Coal; US Geological Survey: Reston, VA, USA, 2006.

22. Finkelman, R.B. Modes of occurrence of environmentally sensitive trace elements in coal. In Environmental Aspects of Trace Elements in Coal; Swaine, D.J., Goodarzi, F., Eds.; Kluwer Academic Publishers: London, UK, 1995; pp. 24-50.

23. Wang, M.S. Study on Environmental Geochemistry of Arsenic in Chinese Coals. Ph.D. Thesis, Institute of Geochemistry, Chinese Academy of Sciences, Guiyang, China, 2005. (In Chinese)

24. Etschmann, B.; Liu, W.H.; Li, K.; Dai, S.F.; Reith, F.; Falconer, D.; Kerr, G.; Paterson, D.; Howard, D.; Kappen, P.; et al. Enrichment of germanium and associated arsenic and tungsten in coal and roll-front uranium deposits. Chem. Geol. 2017, 463, 29-49. [CrossRef]

25. Yudovich, Y.E.; Ketris, M.P. Arsenic in coal: A review. Int. J. Coal Geol. 2005, 61, 141-196. [CrossRef]

26. White, R.N.; Smith, J.V.; Spears, D.A.; Rivers, M.L.; Sutton, S.R. Analysis of iron sulfides from UK coal by synchrotron radiation X-ray fluorescence. Fuel 1989, 68, 1480-1486. [CrossRef]

27. Chen, B.R.; Qian, Q.F.; Yang, Y.N.; Yang, S.J. Concentration distribution of trace element in the 107 samples from coal mines in China. Chin. Sci. Bull. 1985, 1, 27-29. (In Chinese)

28. Xiao, D.X. Research on arsenic in coal and mode of occurrence. In Collected Works of Xi'an Branch of China Coal Research Institute (Volume 3); Shaanxi Science And Technology Press: Xi'an, China, 1989; pp. 36-38. (In Chinese)

29. Ren, D.Y.; Yang, S.J. Distribution characteristics of trace elements in Chinese coal. In Proceedings of the 4th National Anthracology Academic Seminar, Guilin, China; Shaanxi Science and Technology Press: Xi’an, China, 1995. (In Chinese)

30. Zhao, F.H. Study on the Mechanism of Distributions and Occurrences of Hazardous Minor and Trace Elements in Coal and Leaching Experiments of Coal Combustion Residues. Ph.D. Thesis, China University of Mining \& Technology Beijing graduate school, Beijing, China, 1997. (In Chinese)

31. Zhou, Y.P. Distribution Type and Occurrence Form of Arsenic in Anthracite of Laochang Mining Area. Coal Geol. Explor. 1998, 26, 8-13. (In Chinese)

32. Ding, Z.H.; Zheng, B.S.; Zhang, J.; Belkin, H.E.; Finkelman, R.B.; Zhao, F.H.; Zhou, D.X.; Zhou, Y.S.; Chen, C.G. Preliminary results on the geochemistry and mineralogy of arsenic in mineralized coals from Endemic Arsenosis Area in Guizhou Province, China. Sci. China ( Series D) 1999, 29, 421-425. (In Chinese)

33. Niu, S.; Cao, S.; Shen, E. The Status of Arsenic Poisoning in China; Springer: Berlin, Germany, 1997; pp. 78-83.

34. Liu, J.; Zheng, B.S.; Aposhian, H.V.; Zhou, Y.S.; Chen, M.L.; Zhang, A.H.; Waalkes, M.P. Chronic Arsenic Poisoning from Burning High-Arsenic-Containing Coal in Guizhou, China. Environ. Health Perspect. 2002, 110, 119-122. [CrossRef] [PubMed]

35. Jin, Y.L.; Liang, C.K.; He, G.L.; Cao, J.X.; Ma, F.; Wang, H.Z.; Ying, B.; Ji, R.D. Study on distribution of endemic arsenism in China. J. Hyg. Res. 2003, 32, 519-540. (In Chinese)

36. Duker, A.A.; Carranza, E.J.; Hale, M. Arsenic geochemistry and health. Environ. Int. 2005, 31, 631-641. [CrossRef] [PubMed] 
37. Dai, S.F.; Li, D.H.; Ren, D.Y.; Tang, Y.G.; Shao, L.Y.; Song, H.B. Geochemistry of the late Permian No. 30 coal seam, Zhijin Coalfield of Southwest China: Influence of a siliceous low temperature hydrothermal fluid. Appl. Geochem. 2004, 19, 1315-1330. [CrossRef]

38. Dai, S.F.; Ren, D.Y.; Hou, X.Q.; Shao, L.Y. Geochemical and mineralogical anomalies of the late Permian coal in the Zhijin coalfield of southwest China and their volcanic origin. Int. J. Coal Geol. 2003, 55, 117-138. [CrossRef]

39. Ren, D.Y.; Zhao, F.H.; Dai, S.F.; Zhang, J.Y.; Luo, K.L. Geocheminstry of Trace Elements in Coal; Science Press: Beijing, China, 2006; pp. 1-225. (In Chinese)

40. Dai, S.F.; Ren, D.Y.; Chou, C.L.; Finkelman, R.B.; Seredin, V.V.; Zhou, Y.P. Geochemistry of trace elements in Chinese coals: A review of abundances, genetic types, impacts on human health, and industrial utilization. Int. J. Coal Geol. 2012, 94, 3-21. [CrossRef]

41. Finkelman, R.B. Trace and minor elements in coal. In Organic Geochemistry; Engel, M.H., Macko, S.A., Eds.; Springer: New York, NY, USA, 1993; pp. 593-607.

42. Kler, V.; Nenakhova, V.; Saprykin, F.Y. Metallogeny and Geochemistry of Coal- and Shale-Bearing Strata of the USSR: Elements' Buildup Behavior and Its Investigation Techniques; Nauka: Moscow, USSR, Russia, 1988.

43. Kizilshtein, L.Y.; Kholodkov, Y.I. Ecologically hazardous elements in coals of the Donets Basin. Int. J. Coal Geol. 1999, 40, 189-197. [CrossRef]

44. Swaine, D.J.; Goodarzi, F. Environmental Aspects of Trace Elements in Coal; Kluwer Academic Publishers: London, UK, 1995; pp. 1-109.

45. Bouska, V.; Pesek, J. Quality parameters of lignite of the North Bohemian Basin in the Czech Republic in comparison with the world average lignite. Int. J. Coal Geol. 1999, 40, 211-235. [CrossRef]

46. Pesek, J.; Bencko, V.; Sykorova, I.; Vasicek, M.; Michna, O.; Martinek, K. Some trace elements in coal of the Czech Republic, environment and health protection implications. Cent. Eur. J. Public Health 2005, 13, 153-158. [PubMed]

47. US National Committee for Geochemistry. Panel on the trace element geochemistry of coal resource development related to health. In Trace-Element Geochemistry of Coal Resource Development Related to Environmental Quality and Health; National Academy of Sciences: Washington, DC, USA, 1980.

48. Querol, X.; Whateley, M.K.G.; Fernández-Turiel, J.L.; Tuncali, E. Geological controls on the mineralogy and geochemistry of the Beypazari lignite, central Anatolia, Turkey. Int. J. Coal Geol. 1997, 33, 255-271. [CrossRef]

49. Querol, X.; Alastuey, A.; Plana, F.; Lopez-Soler, A.; Tuncali, E.; Toprak, S.; Ocakoglu, F.; Koker, A. Coal geology and coal quality of the Miocene Mugla basin, southwestern Anatolia, Turkey. Int. J. Coal Geol. 1999, 41, 311-332. [CrossRef]

50. Karayigit, A.I.; Gayer, R.A.; Querol, X.; Onacak, T. Contents of major and trace elements in feed coals from Turkish coal-fired power plants. Int. J. Coal Geol. 2000, 44, 169-184. [CrossRef]

51. Palmer, C.A.; Tuncali, E.; Dennen, K.O.; Coburn, T.C.; Finkelman, R.B. Characterization of Turkish coals: A nationwide perspective. Int. J. Coal Geol. 2004, 60, 85-115. [CrossRef]

52. Karayigit, A.I.; Spears, D.A.; Booth, C.A. Antimony and arsenic anomalies in the coal seams from the Gokler coalfield, Gediz, Turkey. Int. J. Coal Geol. 2000, 44, 1-17. [CrossRef]

53. Karayigit, A.I.; Bircan, C.; Mastalerz, M.; Oskay, R.G.; Querol, X.; Lieberman, N.R.; Türkmen, I. Coal characteristics, elemental composition and modes of occurrence of some elements in the İsaalan coal (Balıkesir, NW Turkey). Int. J. Coal Geol. 2017, 172, 43-59. [CrossRef]

54. Sun, J.X.; Jervis, R.E. The trace elements in coal and its distribution in the combustion process. Sci. China (Series A) 1986, 12, 1287-1294. (In Chinese)

55. Dou, T.H.; Xiao, D.X.; Dong, Y.Q.; Zhang, S.A.; Zhang, Q.L. Preliminary Study on the Trace Elements in Coals of Dongsheng Mining District, Shenfu Coalfield. Coal Geol. Explor. 1998, 26, 11-15. (In Chinese)

56. Ren, D.Y.; Zhao, F.H.; Wang, Y.Q.; Yang, S.J. Distributions of minor and trace elements in Chinese coals. Int. J. Coal Geol. 1999, 40, 109-118. [CrossRef]

57. Chen, P.; Huang, W.H.; Tang, X.Y. Features of As content, occurrence in coal and its affect on environment in China. Coal Geol. Explor. 2002, 30,1-4. (In Chinese)

58. Ding, Z.H.; Zheng, B.S.; Long, J.P.; Belkin, H.E.; Finkelman, R.B.; Chen, C.G.; Zhou, D.X.; Zhou, Y.S. Geological and geochemical characteristics of high arsenic coals from endemic arsenosis areas in southwestern Guizhou Province, China. Appl. Geochem. 2001, 16, 1353-1360. [CrossRef] 
59. Zhao, F.H.; Ren, D.Y.; Zheng, B.S.; Hu, T.D.; Liu, T. Research on extension X-ray absorption fine structure spectroscopy of arsenic mode of occurrence in high arsenic coal. Chin. Sci. Bull. 1998, 43, 1549-1551. (In Chinese) [CrossRef]

60. Dai, S.F.; Zeng, R.S.; Sun, Y.Z. Enrichment of arsenic, antimony, mercury, and thallium in a Late Permian anthracite from Xingren, Guizhou, Southwest China. Int. J. Coal Geol. 2006, 66, 217-226. [CrossRef]

61. Nie, A.G.; Xie, H. A study on Emei mantle plume activity and the origin of high-As coal in southwestern Guizhou Province. Chin. J. Geochem. 2006, 25, 238-244. [CrossRef]

62. Dai, S.F.; Wang, P.P.; Ward, C.R.; Tang, Y.G.; Song, X.L.; Jiang, J.H.; Hower, J.C.; Li, T.; Seredin, V.V.; Wagner, N.J.; et al. Elemental and mineralogical anomalies in the coal-hosted Ge ore deposit of Lincang, Yunnan, southwestern China: Key role of $\mathrm{N}_{2}-\mathrm{CO}_{2}$-mixed hydrothermal solutions. Int. J. Coal Geol. 2015, 152, 19-46. [CrossRef]

63. Zhang, J.Y. Enrichment Mechanism and Pollution Restraining of Potentially Hazardous Trace Elements in Coal. Ph.D. Thesis, China University of Mining \& Technology, Beijing, China, 1999. (In Chinese)

64. Wang, Y.; Wang, T.; Kang, G.F.; Lin, Y. A Potential analysis of the availability of coal resources in China. Geol. China 2009, 36, 845-852. (In Chinese)

65. Ge, T. The Features of Harmful Elements in Huainan Coals. Master's Thesis, Anhui University of Science and Technology, Anhui, China, 2009. (In Chinese)

66. Tang, X.Y.; Huang, W.H.; Zhao, J.Y.; Chen, P.; Zhao, Z.G.; Yan, J.P.; Yang, Y.C. Trace Elements in Coal in China; the Commercial Press: Beijing, China, 2004. (In Chinese)

67. Zhao, K.Q.; Wu, S.Z. Study of Distribution and Existence Regularity of Arsenic in Coal, Mengzhuang Coalmine, Huaibei. Geol. Anhui 2010, 20, 34-37. (In Chinese)

68. Chen, J.; Chen, P.; Liu, W.Z. The occurrences and environmental effects of 12 kinds of trace elements in Huainan coal-mining area. Coal Geol. Explor. 2009, 37, 47-52. (In Chinese)

69. Tong, L.H.; Yan, J.P.; Tang, X.Y. Trace Elements and Its distribution characteristic in Coal in Huainan. Min. Saf. Environ. Prot. 2004, 31, 94-96. (In Chinese)

70. Li, H.; Zheng, L.G.; Liu, G.J. The concentration characteristics of trace elements in coal from the Zhangji mining area, Huainan coalfield. Acta Petrol. Mineral. 2011, 30, 696-700. (In Chinese)

71. Chen, P.; Kuang, H.W.; Tang, X.Y. Research on the distribution and occurrence of arsenic in coal. J. China Coal Soc. 2002, 27, 259-263. (In Chinese)

72. Chen, P.; Tang, X.Y. Arsenic in Coal of China. Coal Geol. China 2002, 14, 18-24. (In Chinese)

73. Qian, R.Q.; Yang, X.Y. Multivariable analysis on distribution of potentially hazardous elements in coal seams form Permian Longtan Formation, Anhui Province. Coal Geol. Explor. 2003, 31, 13-16. (In Chinese)

74. Wang, X.B. Geochemistry of Late Triassic coals in the Changhe Mine, Sichuan Basin, southwestern China: Evidence for authigenic lanthanide enrichment. Int. J. Coal Geol. 2009, 80, 167-174. [CrossRef]

75. Luo, K.L.; Li, H.J.; Niu, C.X.; Chen, T.B. Evolution of arsenic and selenium of coals during mass extinction of land species in the Permian in southwestern China and its palaeoenvironmental significance. J. Palaeogeogr. 2008, 10, 385-394. (In Chinese)

76. Dai, S.F.; Wang, X.B.; Chen, W.M.; Li, D.H.; Chou, C.L.; Zhou, Y.P.; Zhu, C.S.; Li, H.; Zhu, X.W.; Xing, Y.W.; et al. A high-pyrite semianthracite of Late Permian age in the Songzao Coalfield, southwestern China: Mineralogical and geochemical relations with underlying mafic tuffs. Int. J. Coal Geol. 2010, 83, 430-445. [CrossRef]

77. Wang, X.B.; Li, D.; Lu, Y.F.; Zhang, Y. Geochemistry of Late Triassic coals from the Changhebian mine in Chongqing, China. Coal Geol. Explor. 2007, 35, 4-9. (In Chinese)

78. Dai, S.F.; Xie, P.P.; Jia, S.H.; Ward, C.R.; Hower, J.C.; Yan, X.Y.; French, D. Enrichment of U-Re-V-Cr-Se and rare earth elements in the Late Permian coals of the Moxinpo Coalfield, Chongqing, China: Genetic implications from geochemical and mineralogical data. Ore Geol. Rev. 2017, 80, 1-17. [CrossRef]

79. Lu, X.H.; Ali, A.; Liu, H.Z.; Zeng, H.C. Multivariable Analysis and Experimental Researches on the Distribution of Trace Elements in Coal. Environ. Chem. 1995, 14, 494-499. (In Chinese)

80. Cui, F.H.; Chen, H.Z. The distribution and occurrence characteristics of arsenic in coal in China. Coal Sci. Technol. 1998, 26, 44-46. (In Chinese)

81. Li, W.H.; Xiong, F.; Jiang, N. Trace Elements in Three Kinds of High Sulphur Coals. Coal Anal. Util. 1993, 1, 7-9. (In Chinese) 
82. Dai, S.F.; Zhang, W.G.; Ward, C.R.; Seredin, V.V.; Hower, J.C.; Li, X.; Song, W.J.; Wang, X.B.; Kang, H.; Zheng, L.C.; et al. Mineralogical and geochemical anomalies of late Permian coals from the Fusui Coalfield, Guangxi Province, southern China: Influences of terrigenous materials and hydrothermal fluids. Int. J. Coal Geol. 2013, 105, 60-84. [CrossRef]

83. Shao, L.Y.; Lu, J.; Tim, J.; Rod, G.; Shang, L.J.; Shen, Z.J.; Zhang, P.F. Mineralogy and geochemistry of the high-organic sulphur coals from the carbonate coal measures of the Late Permian in central Guangxi. J. China Coal Soc. 2006, 31, 770-775. (In Chinese)

84. Dai, S.F.; Zhang, W.G.; Seredin, V.V.; Ward, C.R.; Hower, J.C.; Song, W.J.; Wang, X.B.; Li, X.; Zhao, L.X.; Kang, H.; et al. Factors controlling geochemical and mineralogical compositions of coals preserved within marine carbonate successions: A case study from the Heshan Coalfield, southern China. Int. J. Coal Geol. 2013, 109-110, 77-100. [CrossRef]

85. Dai, S.F.; Xie, P.P.; Ward, C.R.; Yan, X.Y.; Guo, W.M.; French, D.; Graham, I.T. Anomalies of rare metals in Lopingian super-high-organic-sulfur coals from the Yishan Coalfield, Guangxi, China. Ore Geol. Rev. 2017, 88, 235-250. [CrossRef]

86. Zeng, R.S.; Zhao, J.H.; Zhuang, X.G. Quality of Late Permian Coal and Its Controlling Factors in Shuicheng Mining District of Liupanshui Ares, Guizhou. Acta Petrol. Sin. 1998, 14, 549-558. (In Chinese)

87. Zhuang, X.G.; Gong, J.Q.; Wang, Z.Q.; Zeng, R.S.; Xu, W.D. Trace Elements of the Late Permian Coal in the Shuicheng and Liuzhi Coal Fields, Guizhou. Geol. Sci. Technol. Inf. 2001, 20, 53-58. (In Chinese)

88. Ni, J.Y.; Feng, X.B.; Hong, Y.T. The Concentrations of Trace Elements in Coal in Guizhou Province. Environ. Chem. 1998, 17, 339-344. (In Chinese)

89. Zhao, F.H.; Ren, D.Y.; Yin, J.S.; Li, Y.N.; Wang, X.Q. The study on the occurrence of arsenic in coal by sequential chemical extract. China Environ. Sci. 1999, 20, 79-81. (In Chinese)

90. An, D.; He, G.Y.; Hu, X.Q.; Gao, R.Q.; Gao, Z.F. Soot Polluting Poisoning Caused by Arsenic joint Fluorine. Guizhou Med. J. 1992, 16, 1-5. (In Chinese)

91. Zhou, D.X.; Liu, D.N.; Zhu, S.L.; Li, B.L.; Jin, D.X.; Zhou, Y.S.; Lu, X.Z.; Ha, X.B.; Zhou, C. Investigation of Chronic Arsenic Poisoning Caused by High Arsenic Coal Pollution. Chin. J. Prev. Med. 1993, 27, 147-150. (In Chinese)

92. Dai, S.F.; Chou, C.L.; Yue, M.; Luo, K.L.; Ren, D.Y. Mineralogy and geochemistry of a Late Permian coal in the Dafang Coalfield, Guizhou, China: Influence from siliceous and iron-rich calcic hydrothermal fluids. Int. J. Coal Geol. 2005, 61, 241-258. [CrossRef]

93. Feng, X.B.; Ni, J.Y.; Hong, Y.T.; Zhu, J.M.; Zhou, B.; Wang, Y. A preliminary study on the distribution laws of some volatile trace elements in coal of Guizhou province. Environ. Chem. 1998, 17, 148-153. (In Chinese)

94. Dai, S.F.; Ren, D.Y.; Tang, Y.G.; Yue, M.; Hao, L.M. Concentration and distribution of elements in Late Permian coals from western Guizhou Province, China. Int. J. Coal Geol. 2005, 61, 119-137. [CrossRef]

95. Lei, J.J. The Occurrence Regularity of Guizhou Late Permian of Sulfur in Coal—And Structure, Composition and Origin of High Organic Sulfur Coal. Ph.D. Thesis, China University of Mining \& Technology, Beijing, China, 1993. (In Chinese)

96. Li, D.H.; Tang, Y.G. Geological Genesis of Coal Geochemical Anomalies of the Late Permian Coals from the Qinglong Coalfield in Western Guizhou, China. Geol. Rev. 2005, 51, 163-168. (In Chinese)

97. Guo, Y.T.; Wang, Y.B.; Fang, A.M. The harmful trace elements and its distribution in coal seams of the Late Permian in west of Guizhou. In Collected Works of Coal Geology Research; China Industry Publishing House: Beijing, China, 1996; pp. 188-194. (In Chinese)

98. Dai, S.F.; Seredin, V.V.; Ward, C.R.; Hower, J.C.; Xing, Y.W.; Zhang, W.G.; Song, W.J.; Wang, P.P. Enrichment of U-Se-Mo-Re-V in coals preserved within marine carbonate successions: Geochemical and mineralogical data from the Late Permian Guiding Coalfield, Guizhou, China. Miner. Deposita 2015, 50, 159-186. [CrossRef]

99. Yang, J.Y. Contents and occurrence modes of trace elements in the Late Permian coals from Puan Coalfield, Guizhou Province. J. Fuel Chem. Technol. 2006, 34, 129-135. (In Chinese)

100. Zhuang, X.G.; Yang, S.K.; Zeng, R.S.; Xu, W.D. Characteristics of Trace Elements in Coals from Several Main Coal Districts in China. Geol. Sci. Technol. Inf. 1999, 18, 63-66. (In Chinese)

101. Tang, Y.G.; Yin, Z.R.; Chang, C.X.; Zhang, Y.Z.; Song, H.B.; Wang, S.Q.; Hao, L. Distribution of trace elements in the Kailuan coalfield. J. China Coal Soc. 2005, 30, 80-84. (In Chinese)

102. Dai, S.F. Geological-Geochemical Behaviors and Enrichment Models of Associated Elements in Coal. Ph.D. Thesis, China University of Mining \& Technology, Beijing, China, 2002. (In Chinese) 
103. Dai, S.F.; Ren, D.Y. Effects of Magmatic Intrusion on Mineralogy and Geochemistry of Coals from the Fengfeng-Handan Coalfield, Hebei, China. Energy Fuels 2007, 21, 1663-1673. [CrossRef]

104. Yuan, S.W. China Coal Quality Review; China Industry Publishing House: Beijing, China, 1999. (In Chinese)

105. Wu, Z.Y.; Li, Y.H.; Zhou, Y.C. Geochemical behavior of trace elements in coals in Baishan area, Jilin Province. Coal Geol. Explor. 2004, 32, 8-10. (In Chinese)

106. Zhou, X.D. Study on change of the arsenic content in coal area of Middle Jiangxi Province. Coal Geol. China 1991, 3, 39-44. (In Chinese)

107. Zhang, Z.F.; Fan, J.C.; Jin, J.F.; Yang, Y.C.; Song, L.J.; Yang, H. Mode of Occurrence of Pb, As, Be, Cr in Coal. J. Fuel Chem. Technol 20, 206-212. (In Chinese)

108. Ren, D.Y.; Xu, D.W.; Zhang, J.Y.; Zhao, F.H.; Li, G.Z.; Xie, L.W. Distribution of Associated Elements in Coals from Shenbei Coalfield. J. China Univ. Min. Technol. 1999, 28, 5-8. (In Chinese)

109. Querol, X.; Alastuey, A.; Lopez-Soler, A.; Plana, F.; Fernandez-Turiel, J.L.; Zeng, R.S.; Xu, W.D.; Zhuang, X.G.; Spiro, B. Geological controls on the mineral matter and trace elements of coals from the Fuxin basin, Liaoning Province, northeast China. Int. J. Coal Geol. 1997, 34, 89-109. [CrossRef]

110. Wang, W.F.; Qin, Y.; Song, D.Y. Cleaning potential of hazardous elements during coal washing. J. Fuel Chem. Technol. 2003, 32, 295-299. (In Chinese)

111. Li, S.S. Trace Element Geochemistry of Late Paleozoic Coals in the Eastern Margin of Ordos Basin. Ph.D. Thesis, China University of Mining \& Technology, Beijing, China, 2005. (In Chinese)

112. Dai, S.F.; Zou, J.H.; Jiang, Y.F.; Ward, C.R.; Wang, X.B.; Li, T.; Xue, W.F.; Liu, S.D.; Tian, H.M.; Sun, X.H.; et al. Mineralogical and geochemical compositions of the Pennsylvanian coal in the Adaohai Mine, Daqingshan Coalfield, Inner Mongolia, China: Modes of occurrence and origin of diaspore, gorceixite, and ammonian illite. Int. J. Coal Geol. 2012, 94, 250-270. [CrossRef]

113. Dai, S.F.; Li, T.J.; Jiang, Y.F.; Ward, C.R.; Hower, J.C.; Sun, J.H.; Liu, J.J.; Song, H.J.; Wei, J.P.; Li, Q.Q.; et al. Mineralogical and geochemical compositions of the Pennsylvanian coal in the Hailiushu Mine, Daqingshan Coalfield, Inner Mongolia, China: Implications of sediment-source region and acid hydrothermal solutions. Int. J. Coal Geol. 2015, 137, 92-110. [CrossRef]

114. Dai, S.F.; Li, D.; Chou, C.L.; Zhao, L.; Zhang, Y.; Ren, D.Y.; Ma, Y.W.; Sun, Y.Y. Mineralogy and geochemistry of boehmite-rich coals: New insights from the Haerwusu Surface Mine, Jungar Coalfield, Inner Mongolia, China. Int. J. Coal Geol. 2008, 74, 185-202. [CrossRef]

115. Yang, N.; Tang, S.H.; Zhang, S.H.; Chen, Y.Y. Geochemistry of trace elements in the No. 5 Coal from the Chuancaogedan Mine, Junger Coalfield. Earth Sci. Front. 2016, 23, 74-82. (In Chinese)

116. Dai, S.F.; Ren, D.Y.; Chou, C.L.; Li, S.S.; Jiang, Y.F. Mineralogy and geochemistry of the No. 6 Coal (Pennsylvanian) in the Junger Coalfield, Ordos Basin, China. Int. J. Coal Geol. 2006, 66, 253-270. [CrossRef]

117. Dai, S.F.; Zhao, L.; Peng, S.P.; Chou, C.L.; Wang, X.B.; Zhang, Y.; Li, D.; Sun, Y.Y. Abundances and distribution of minerals and elements in high-alumina coal fly ash from the Jungar Power Plant, Inner Mongolia, China. Int. J. Coal Geol. 2010, 81, 320-332. [CrossRef]

118. Bai, X.F. The Distributions, Modes of Occurrence and Volatility of Trace Elements in Coals of China. Ph.D. Thesis, China Coal Research Institute, Beijing, China, 2003. (In Chinese)

119. Li, H.M.; Fei, S.Y.; Wang, S.J.; Zhang, J.Q.; Cao, Y.R.; Lei, Z.M.; Liu, X.W. Inorganic Geochemistry of Coal in Coal-Bearing Strata in Erdos Basin of Middle Jurassic; Geological Publishing House: Beijing, China, 1993. (In Chinese)

120. Dai, S.F.; Liu, J.J.; Ward, C.R.; Hower, J.C.; Xie, P.P.; Jiang, Y.F.; Hood, M.M.; O’Keefe, J.M.K.; Song, H.J. Petrological, geochemical, and mineralogical compositions of the low-Ge coals from the Shengli Coalfield, China: A comparative study with Ge-rich coals and a formation model for coal-hosted Ge ore deposit. Ore Geol. Rev. 2015, 71, 318-349. [CrossRef]

121. Song, D.Y. The Combustion-Leaching and Migration and Its Environmental Effect of Harmful Trace Elements in Coals. Ph.D. Thesis, China University of Mining \& Technology, Xuzhou, China, 2003. (In Chinese)

122. Dai, S.F.; Hower, J.C.; Ward, C.R.; Guo, W.M.; Song, H.J.; O’Keefe, J.M.K.; Xie, P.P.; Hood, M.M.; Yan, X.Y. Elements and phosphorus minerals in the middle Jurassic inertinite-rich coals of the Muli Coalfield on the Tibetan Plateau. Int. J. Coal Geol. 2015, 144-145, 23-47. [CrossRef]

123. Zeng, R.S.; Zhuang, X.G.; Yang, S.K. Coal quality characteristics in the middle part of coal bearing area in west of Shandong. Coal Geol. China 2000, 12, 10-15. (In Chinese) 
124. Hu, Z.X. Study on Some Sensitive Trace Elements and Their Environmental Significance in Coal from the Jibei Coalfield. Ph.D. Thesis, China University of Mining \& Technology, Xuzhou, China, 2009. (In Chinese)

125. Liu, G.J. Study on Environmental Geochemistry of Trace Elements in Coal in Yanzhou Mining Area. Ph.D. Thesis, China University of Mining \& Technology, Beijing, China, 1999. (In Chinese)

126. Zhang, H.Y. Study on the Enrichment Characteristics of Sulfur and Trace Elements in Coal. Ph.D. Thesis, China University of Mining \& Technology, Beijing, China, 2001. (In Chinese)

127. Liu, G.J.; Yang, P.; Peng, Z.C.; Wang, G.L. Geochemistry of trace elements from the No. 3 coal seam of Shanxi Formation in the Yanzhou mining district. Geochimica 2003, 32, 255-262. (In Chinese)

128. Zhuang, X.G.; Zeng, R.S.; Xu, W.D. Trace Elements in 9 Coal from Antaibao Open Pit Mine, Pingshuo, Shanxi Province. Earth Sci. 1998, 23, 583-588. (In Chinese)

129. Ge, Y.T. Trace elements in coal gangue and its influence on environment in Shanxi. Coal Geol. China 1996, 8, 58-62. (In Chinese)

130. Wang, Y.Q. Study on the Distribution and Occurrence Characteristics of Trace Elements in Coal and Its Combustion Products. Ph.D. Thesis, China University of Mining \& Technology, Beijing, China, 1994. (In Chinese)

131. Zhang, J.Y.; Zheng, C.G.; Ren, D.Y.; Chou, C.L.; Liu, J.; Zeng, R.S.; Wang, Z.P.; Zhao, F.H.; Ge, Y.T. Distribution of potentially hazardous trace elements in coals from Shanxi province, China. Fuel 2004, 83, 129-135. [CrossRef]

132. Lu, X.W. A preliminary study on the arsenic content, distribution laws and speciations of coal in Weibei Area, Shaanxi Province. J. Arid Land Resour. Environ. 2003, 17, 66-70. (In Chinese)

133. Wang, X.B.; Dai, S.F.; Ren, D.Y.; Yang, J.Y. Mineralogy and geochemistry of Al-hydroxide/oxyhydroxide mineral-bearing coals of Late Paleozoic age from the Weibei coalfield, southeastern Ordos Basin, North China. Appl. Geochem. 2011, 26, 1086-1096. [CrossRef]

134. Dai, S.F.; Luo, Y.B.; Seredin, V.V.; Ward, C.R.; Hower, J.C.; Zhao, L.; Liu, S.D.; Zhao, C.L.; Tian, H.M.; Zou, J.H. Revisiting the late Permian coal from the Huayingshan, Sichuan, southwestern China: Enrichment and occurrence modes of minerals and trace elements. Int. J. Coal Geol. 2014, 122, 110-128. [CrossRef]

135. Dai, S.F.; Liu, J.J.; Ward, C.R.; Hower, J.C.; French, D.; Jia, S.H.; Hood, M.M.; Garrison, T.M. Mineralogical and geochemical compositions of Late Permian coals and host rocks from the Guxu Coalfield, Sichuan Province, China, with emphasis on enrichment of rare metals. Int. J. Coal Geol. 2016, 166, 71-95. [CrossRef]

136. Fu, X.G.; Wang, J.; Tan, F.W.; Feng, X.L.; Zeng, S.Q. Minerals and potentially hazardous trace elements in the Late Triassic coals from the Qiangtang Basin, China. Int. J. Coal Geol. 2013, 116-117, 93-105. [CrossRef]

137. Dai, S.F.; Yang, J.Y.; Ward, C.R.; Hower, J.C.; Liu, H.D.; Garrison, T.M.; French, D.; O'Keefe, J.M.K. Geochemical and mineralogical evidence for a coal-hosted uranium deposit in the Yili Basin, Xinjiang, northwestern China. Ore Geol. Rev. 2015, 70, 1-30. [CrossRef]

138. Gu, D.J. Distribution, Migration and Enrichment of Trace Elements in Tertiary Lignite of Yunnan. Ph.D. Thesis, China University of Mining \& Technology Beijing graduate school, Beijing, China, 1990. (In Chinese)

139. Zhou, Y.P. Distribution and controlling factors of arsenic in some coals in Yunnan. Coal Geol. Explor. 1983, 11, 2-8. (In Chinese)

140. Dai, S.F.; Ren, D.Y.; Zhou, Y.P.; Chou, C.L.; Wang, X.B.; Zhao, L.; Zhu, X.W. Trace Elements and Minerals Enriched in Coals by the Composite Origin of Synsedimentary Volcanic Ash and Submarine Spouting. Chin. Sci. Bull. 2008, 53, 3120-3126. (In Chinese)

141. Dai, S.F.; Tian, L.W.; Chou, C.L.; Zhou, Y.P.; Zhang, M.Q.; Zhao, L.; Wang, J.M.; Yang, Z.; Cao, H.Z.; Ren, D.Y. Mineralogical and compositional characteristics of Late Permian coals from an area of high lung cancer rate in Xuan Wei, Yunnan, China: Occurrence and origin of quartz and chamosite. Int. J. Coal Geol. 2008, 76, 318-327. [CrossRef]

142. Dai, S.F.; Ren, D.Y.; Zhou, Y.P.; Chou, C.L.; Wang, X.B.; Zhao, L.; Zhu, X.W. Mineralogy and geochemistry of a superhigh-organic-sulfur coal, Yanshan Coalfield, Yunnan, China: Evidence for a volcanic ash component and influence by submarine exhalation. Chem. Geol. 2008, 255, 182-194. [CrossRef]

143. Dai, S.F.; Li, T.; Seredin, V.V.; Ward, C.R.; Hower, J.C.; Zhou, Y.P.; Zhang, M.Q.; Song, X.L.; Song, W.J.; Zhao, C.L. Origin of minerals and elements in the Late Permian coals, tonsteins, and host rocks of the Xinde Mine, Xuanwei, eastern Yunnan, China. Int. J. Coal Geol. 2014, 121, 53-78. [CrossRef]

144. Dai, S.F.; Han, D.X.; Chou, C.L. Petrography and geochemistry of the Middle Devonian coal from Luquan, Yunnan Province, China. Fuel 2006, 85, 456-464. [CrossRef] 
145. Wang, D.Y. Research on Arsenic content in the coal, distribution characteristics and classification. Coal Qual. Technol. 2000, 6, 27-30. (In Chinese)

146. Zheng, L.G.; Liu, G.J.; Chou, C.L.; Gao, L.F.; Peng, Z.C. Arsenic in Chinese Coals: Its abundance, distribution, modes of occurrence, enrichment processes, and environmental significance. Acta Geosci. Sin. 2006, 27, 355-366. (In Chinese)

147. Lv, S.; Ma, S.M.; Kong, X.R.; Sun, M.M.; Zhao, M.D.; Guo, C.X. Study on the distribution and gradation of arsenic in Chinese coals. China Min. Mag. 2014, 23, 125-129. (In Chinese)

148. Li, D.H.; Chen, K.; Deng, T.; Cheng, F.P.; Yang, J. Distribution and enrichment factors of arsenic in coals of Southwestern China. J. China Univ. Min. Technol. 2002, 31, 419-422. (In Chinese)

149. Xie, H. Study on composition of high arsenic coal in Western Guizhou. Coal Eng. 2010, 4, 87-89. (In Chinese)

150. Liu, B.; Huang, W.H.; Ao, W.H.; Yan, D.Y.; Xu, Q.L.; Teng, J. Geochemistry characteristics of sulfur and its effect on hazardous elements in the Late Paleozoic coal from the Qinshui Basin. Earth Sci. Front. 2016, 23, 59-67. (In Chinese)

151. Chen, J.; Chen, P.; Yao, D.X.; Liu, Z.; Wu, Y.S.; Liu, W.Z.; Hu, Y.B. Mineralogy and geochemistry of Late Permian coals from the Donglin Coal Mine in the Nantong coalfield in Chongqing, southwestern China. Int. J. Coal Geol. 2015, 149, 24-40. [CrossRef]

152. Zhao, F.H.; Ren, D.Y.; Peng, S.P.; Wang, Y.Q.; Zhang, J.Y.; Ding, Z.H.; Cong, Z.Y. The modes of occurrence of arsinic in coal. Adv. Earth Sci. 2003, 18, 214-220. (In Chinese)

153. Wang, Y.Q.; Meng, H.T.; Ren, D.Y. Characteristics and washability evaluation of pyrite in the Wangtaipu Coal Mine of Jincheng. In Collected Works of Coalfield Geology Research; China Industry Publishing House: Beijing, China, 1996; pp. 180-187. (In Chinese)

154. Huang, W.H.; Yang, Q.; Tang, D.Z.; Kang, X.D.; Liu, D.M. Trace elements geochemistry of the coals in the Taiyuan Formation from Zaozhuang Coal Field. Geoscience 2000, 14, 61-68. (In Chinese)

155. Zhang, Z.F.; Fan, J.C. The forms of combination of As, Pb, Cr in Xiaolongtan Brown Coal. Coal Convers. 1993, 16, 86-89. (In Chinese)

156. Guo, X.; Zheng, C.G.; Liu, Y.H.; Liu, J.; Lu, X.H. The study on the mode of occurrence of mercury, arsenic and selenium in coal. J. Eng. Thermophys. 2001, 22, 763-766. (In Chinese)

157. Nie, A.G.; Xie, H. A research on origin between Emei Mountain Basalt Magma and high-As coal in Guizhou. Coal Geol. Explor. 2004, 32, 8-10. (In Chinese)

158. Wang, Y.Q.; Zhang, R.G.; Wang, L.P.; Ren, D.Y.; Zhao, F.H. Extract experiments of minor and trace element occurrence in coal. Coal Geol. China 1997, 9, 23-25. (In Chinese)

159. Liu, G.J.; Yang, P.; Peng, Z.C.; Wang, G.L.; Cao, Z.H. Occurrence of trace elements in coal of Yanzhou Mining District. Geochimica 2002, 31, 85-90. (In Chinese)

160. Ding, Z.H.; Zheng, B.S.; Finkelmam, R B.; Hu, T.D.; Zhou, Y.S. Subsequent leaching study of typical High-As coal samples from Southwest Guizhou Province. Earth Sci. 2003, 28, 209-213. (In Chinese)

161. Ding, Z.H. Study on Mineralogy and Geochemistry of High-As Coals from Guizhou Province, China. Ph.D. Thesis, Institute of Geochemistry, Chinese Academy of Sciences, Guizhou, China, 2000. (In Chinese)

(C) 2017 by the authors. Licensee MDPI, Basel, Switzerland. This article is an open access article distributed under the terms and conditions of the Creative Commons Attribution (CC BY) license (http://creativecommons.org/licenses/by/4.0/). 